\title{
TOPSIS Method Based on Correlation Coefficient and Entropy Measure for Linguistic Pythagorean Fuzzy Sets and Its Application to Multiple Attribute Decision Making
}

\author{
Mingwei Lin $\left(\mathbb{D},{ }^{1,2,3} \text { Chao Huang }(\mathbb{D})^{1,2} \text { and Zeshui Xu }{ }^{4}\right)^{4}$ \\ ${ }^{1}$ College of Mathematics and Informatics, Fujian Normal University, Fuzhou, Fujian 350117, China \\ ${ }^{2}$ Digital Fujian Internet-of-Things Laboratory of Environmental Monitoring, Fujian Normal University, Fuzhou, \\ Fujian 350117, China \\ ${ }^{3}$ Digital Fujian Institute of Big Data for Agriculture and Forestry, Fujian Agriculture and Forestry University, Fuzhou, \\ Fujian 350002, China \\ ${ }^{4}$ Business School, Sichuan University, Chengdu, Sichuan 610064, China
}

Correspondence should be addressed to Mingwei Lin; linmwcs@163.com

Received 7 May 2019; Revised 27 September 2019; Accepted 8 October 2019; Published 30 October 2019

Academic Editor: Roberto Natella

Copyright ( 2019 Mingwei Lin et al. This is an open access article distributed under the Creative Commons Attribution License, which permits unrestricted use, distribution, and reproduction in any medium, provided the original work is properly cited.

The linguistic Pythagorean fuzzy set (LPFS) is an important implement for modeling the uncertain and imprecise information. In this paper, a novel TOPSIS (Technique for Order of Preference by Similarity to Ideal Solution) method is proposed for LPFSs based on correlation coefficient and entropy measure. To this end, the correlation coefficient is proposed for the relationship measurement between LPFSs. Afterwards, two entropy measures are developed to calculate the attribute weight information. Then, a novel linguistic Pythagorean fuzzy TOPSIS (LPF-TOPSIS) method is proposed to solve multiple attribute decisionmaking problems. Finally, the LPF-TOPSIS method is applied to handle a case concerning the selection of firewall productions, and then, a case concerning the security evaluation of computer systems is given to conduct the comparative analysis between the proposed LPF-TOPSIS method and previous decision-making methods for validating the superiority of the proposed LPFTOPSIS method.

\section{Introduction}

The multiple attribute decision making (MADM) refers to the process in which some alternatives with respect to multiple attributes are rated and ranked [1-5]. The multiple attribute decision-making processes happen in various fields [6-9]. In the initial stage of decision-making evolution, crisp numbers are usually used to rate the alternatives [10]. With the growing complexity of decision-making problems, human beings do not have the capability to express the vague and ambiguous information [11-13]. To model this kind of information, fuzzy set (FS) was initially put forward in [14]. The FS only expresses the membership degree (MD), but it does not model the dissenting opinions, namely, nonmembership degree (NMD). To overcome this defect, the concept of intuitionistic fuzzy sets (IFSs) [15] was proposed, which is composed of MD and NMD. The sum of MD and $\mathrm{NMD}$ in an IFS should satisfy that $\mathrm{MD}+\mathrm{NMD} \leq 1$. To improve the modeling capability, Pythagorean fuzzy set (PFS) $[16,17]$ was put forward by Yager et al., in which the $\mathrm{MD}$ and the NMD must satisfy that $\mathrm{MD}^{2}+\mathrm{NMD}^{2} \leq 1$. Because of its superiority, the concept of PFS has attracted much attention from researchers [18-22]. It has been widely applied to various fields, such as pattern recognition [23,24], medical diagnosis [25, 26], risk attitudinal assignment [27], and fault diagnosis [28].

In some situations, qualitative terms are preferred to quantify the evaluation information of the objects [29-33]. A fuzzy linguistic approach was proposed by Zadeh [34]. It can model the evaluation information of decision makers (DMs) 
as the qualitative form $[35,36]$. To extend the modeling capability of IFSs into the qualitative setting, Zhang [37] proposed the linguistic intuitionistic fuzzy set (LIFS), which is produced by combining IFS with linguistic term set (LTS) [38-40]. In the LIFSs, each linguistic intuitionistic fuzzy number (LIFN) consists of MD and NMD whose values are expressed using the linguistic terms in the LTS. The MD and NMD should satisfy that $I(\mathrm{MD})+I(\mathrm{NMD}) \leq \tau$, where $I(\mathrm{MD})$ and $I$ (NMD) denote the subscripts of MD and NMD and $\tau$ is the cardinality of LTS. Inspired by the LIFS, Garg put forward the linguistic Pythagorean fuzzy sets (LPFSs) [41], in which each linguistic Pythagorean fuzzy number (LPFN) also uses two linguistic terms to express its MD and NMD. The main difference between LPFS and LIFS is that the square sum of the subscripts of MD and NMD in the LPFN is less than or equal to the square of the cardinality of the LTS. As shown in Figure 1, the distribution area of the LPFN is larger than that of the LIFN. The LIFN may only fall into the blue area, while the LPFN may fall into the blue area or yellow area. Thus, an LIFN is also an LPFN, while an LPFN may not be an LIFN. It indicates that the LPFS can provide DMs with more freedom to express their information. It has drawn the attention of researchers. For example, Lin et al. [42] put forward some novel interactional operational laws for computing LPFSs, based on which, the partitioned Bonferroni mean was extended for fusing LPFSs.

As for the MADM methods for LPFSs, there exist some deficiencies that are analyzed as follows:

(1) There exist few MADM methods that have been proposed to handle LPFSs. Until now, only Garg [41] and Lin et al. [42] proposed some aggregation operators for fusing LPFSs so as to rank the alternatives. The aggregation operators are noncompensatory MADM methods, which simply aggregate all the attribute values into a single value and cannot achieve trade-offs among conflicting attributes.

(2) The TOPSIS method [43-45] is a classical tool used to solve MADM problems. It belongs to the compensatory MADM methods. It has been extended to solve IFSs [46], interval-valued IFSs [47, 48], and PFSs [49-51]. However, the data structure contained in LPFSs is different from that of the previous fuzzy information. Therefore, the previous TOPSIS methods cannot be directly used for LPFSs. Moreover, to the best of our knowledge, there are no research results that report the use of the TOPSIS method in the LPFSs.

(3) The classical TOPSIS method uses the distance measure to get ideal solutions [52]. However, the TOPSIS method based on distance measure cannot distinguish ideal solutions in some special cases [53]. It is necessary to improve the TOPSIS method using other measure, such as correlation coefficient. Moreover, the attribute weight information in the MADM problems is usually unknown. Thus, how to determine the attribute weight information is also a big challenge.
To overcome the above deficiencies, we propose a novel TOPSIS method for LPFSs, which combines the correlation coefficient and entropy measure. Our contributions can be summarized as follows:

(1) We propose the equations of correlation coefficient and weighted correlation coefficient for LPFSs based on MD, NMD, and hesitance degree, and then their properties are discussed.

(2) Two entropy measures are developed to measure the uncertainty degree of LPFSs considering MD and NMD, and then their properties are discussed. These entropy measures are used to derive the attribute weight information for the MADM problems under the LPFS environment.

(3) The proposed correlation coefficient and entropy measure are used to modify the classical TOPSIS method for proposing a novel TOPSIS method for LPFSs, which is called LPF-TOPSIS. The LPFTOPSIS method is used to solve the MADM problems with unknown attribute weight information.

(4) A case concerning the selection of firewall productions is studied to demonstrate the application of the proposed LPF-TOPSIS method. Then, a case concerning the security evaluation of computer systems is provided to compare the proposed LPFTOPSIS method with the existing decision-making methods based on aggregation operators [41, 42].

Our study is different from the existing studies [41, 42] in the following aspects:

(1) The attribute weight information in the existing studies is given by DMs in advance, while in our study, the attribute weight information is determined using entropy measures of LPFSs.

(2) The correlation coefficient and entropy measure have not been developed for LPFSs in the existing studies. Both of them are achieved in our study, and their properties are discussed. Therefore, our study can help to enrich the theoretical basis of LPFSs and promote their wide applications in various fields.

(3) The aggregator operators in the existing studies are noncompensatory MADM methods, which are inefficient to solve MADM problems with conflicting attributes. The proposed LPF-TOPSIS method in our study is a compensatory MADM method. It can efficiently achieve trade-offs among conflicting attributes.

The remainder of this paper is organized as follows: Section 2 provides some basic knowledge about LPFSs and classical TOPSIS method. The correlation coefficient and two entropy measures are devised for LPFSs in Section 3. A novel LPF-TOPSIS method based on correlation coefficient and entropy measure is developed in Section 4. A case concerning the selection of firewall productions is provided and comparative analyses are performed in Section 5. Finally, some valuable conclusions are drawn in Section 6. 


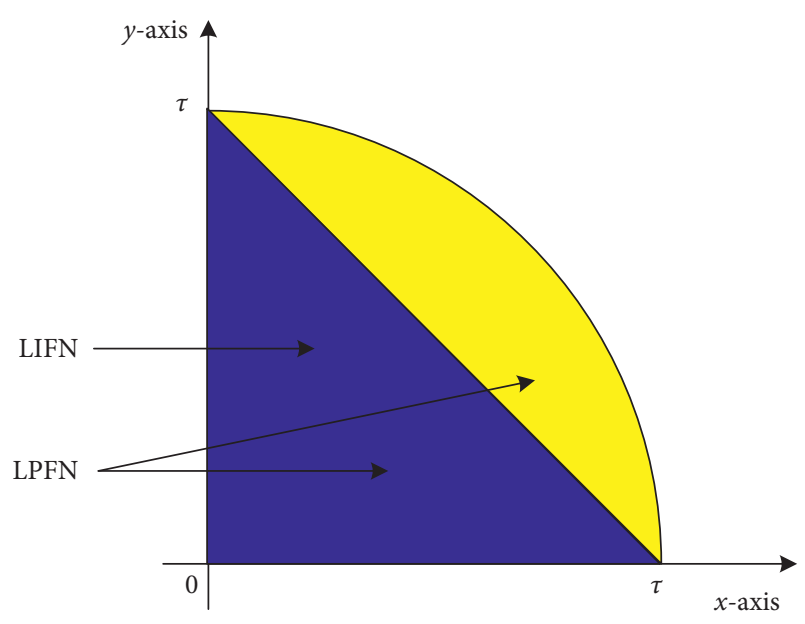

FIgURE 1: The distribution areas of LIFN and LPFN.

\section{Preliminaries}

In this section, we briefly review some basic knowledge about LPFSs and classical TOPSIS method.

2.1. Linguistic Pythagorean Fuzzy Sets. The LPFS was proposed by Garg [41] by combining PFS with LTS. Its definition is given as follows.

Definition 1 (see [41]). Given a reference set $X=\left\{x_{1}, x_{2}, \ldots\right.$, $\left.x_{n}\right\}$ and a continuous LTS $S=\left\{s_{\beta} \mid \beta \in[0, \tau]\right\}$ with a positive integer $\tau$, then the mathematical form of an LPFS on $X$ is defined as

$$
A=\left\{\left\langle x, s_{p}(x), s_{q}(x)\right\rangle \mid x \in X\right\},
$$

where $s_{p}(x)$ and $s_{q}(x)$ are two functions, which return the MD and NMD of the element $x$ belonging to the LPFS $A$. Each pair of MD and NMD $\left(s_{p}(x), s_{q}(x)\right)$ is simplified as $\alpha=\left(s_{p}, s_{q}\right)$, which is named an linguistic Pythagorean fuzzy number (LPFN). In the LPFNs, the MD $s_{p}$ and NMD $s_{q}$ should satisfy that $0 \leq p \leq \tau, 0 \leq q \leq \tau$, and $p^{2}+q^{2} \leq \tau^{2}$. Let $s_{h}=s \sqrt{\tau^{2}-p^{2}-q^{2}}$, then $s_{h}$ is named as the hesitance degree (HD) of this LPFN.

Garg [41] also put forward a comparison method consisting of score function and accuracy function to compare LPFNs.

Definition 2 (see [41]). Given an LPFN $A=\left(s_{p}, s_{q}\right)$ with $s_{p}$ and $s_{q}$ being the elements of a continuous LTS $S=\left\{s_{\beta} \mid \beta \in[0, \tau]\right\}$, then the score function is defined as $S(A)=s \sqrt{\left(\tau^{2}+p^{2}-q^{2}\right) / 2}$ and its accuracy function is defined as $H(A)=s \sqrt{p^{2}+q^{2}}$.

Suppose that there are two LPFNs $A$ and $B$, if $S(A)>S(B)$, then it can be considered as $A>B$. If $S(A)=S(B)$, then their accuracy function values should be further compared. If $H(A)>H(B)$, then $A>B$. If $H(A)=H(B)$, then $A=B$.

To aggregate LPFNs $A_{i}=\left(s_{p_{i}}, s_{q_{i}}\right), i=1,2, \ldots, n$, Garg [41] devised the linguistic Pythagorean fuzzy weighted averaging (LPFWA) and linguistic Pythagorean fuzzy weighted geometric (LPFWG) aggregation operators as follows.

Definition 3 (see [41]). Given LPFNs $A_{i}=\left(s_{p_{i}}, s_{q_{i}}\right), i=$ $1,2, \ldots, n$ in which $s_{p}$ and $s_{q}$ belong to a continuous LTS $S=\left\{s_{\beta} \mid \beta \in[0, \tau]\right\}$, then the LPFWA aggregation operator is a function that is defined as

$$
\begin{aligned}
& \operatorname{LPFWA}\left(A_{1}, A_{2}, \ldots, A_{n}\right)=\omega_{1} A_{1} \oplus \omega_{2} A_{2} \oplus \ldots \oplus \omega_{n} A_{n}
\end{aligned}
$$

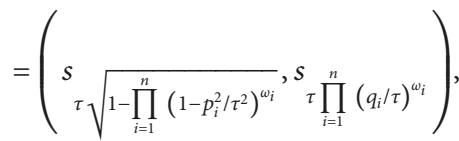

where $\omega=\left(\omega_{1}, \omega_{2}, \ldots, \omega_{n}\right)$ denotes the weight vector of these LPFNs, which satisfies that $0 \leq \omega_{j} \leq 1$ and $\sum_{j=1}^{n} \omega_{j}=1$.

Definition 4 (see [41]). Given LPFNs $A_{i}=\left(s_{p_{i}}, s_{q_{i}}\right), i=$ $1,2, \ldots, n$ in which $s_{p}$ and $s_{q}$ belong to a continuous LTS $S=\left\{s_{\beta} \mid \beta \in[0, \tau]\right\}$, then the LPFWG aggregation operator is a function that is defined as

$$
\begin{aligned}
& \operatorname{LPFWG}\left(A_{1}, A_{2}, \ldots, A_{n}\right)=\omega_{1} A_{1} \otimes \omega_{2} A_{2} \otimes \ldots \otimes \omega_{n} A_{n} \\
& =\left(s \prod_{i=1}^{n}\left(p_{i} / \tau\right)^{\omega_{i}}, s \sqrt{1-\prod_{i=1}^{n}\left(1-q_{i}^{2} / \tau^{2}\right)^{\omega_{i}}}\right),
\end{aligned}
$$

where $\omega=\left(\omega_{1}, \omega_{2}, \ldots, \omega_{n}\right)$ denotes the weight vector of these LPFNs, which satisfies that $0 \leq \omega_{j} \leq 1$ and $\sum_{j=1}^{n} \omega_{j}=1$.

2.2. Classical TOPSIS Method. The TOPSIS is a classical MADM method based on ideal solutions [54]. Its designing idea is that the optimal alternative should be closest to the positive ideal solution (PIS) and furthest away from the negative ideal solution (NIS) [55]. There is an MADM problem composed of $m$ alternatives $c_{i}, i=$ $1,2, \ldots, m$ and $n$ attributes $u_{j}, j=1,2, \ldots, n$. All the evaluation information of the alternatives concerning their attributes compose a decision matrix $X=\left(x_{i j}\right)_{m \times n}$. The weight vector of attributes is $\omega=\left(\omega_{1}, \omega_{2}, \ldots, \omega_{n}\right)^{T}$. It satisfies $0 \leq \omega_{j} \leq 1$ and $\sum_{j=1}^{n} \omega_{j}=1$. The decision-making process of the classical TOPSIS method can be summarized as follows:

Step 1: normalize the decision matrix $X=\left(x_{i j}\right)_{m \times n}$ to be $R=\left(r_{i j}\right)_{m \times n}$ using

$$
r_{i j}=\frac{x_{i j}-\min _{i} x_{i j}}{\max _{i} x_{i j}-\min _{i} x_{i j}}, \quad i=1,2, \ldots, m ; j=1,2, \ldots, n .
$$

Step 2: determine the PIS and NIS as 


$$
\begin{aligned}
R^{+}= & \left(r_{1}^{+}, r_{2}^{+}, \ldots, r_{n}^{+}\right) \\
& =\left(\max _{1 \leq i \leq m}\left\{r_{i j}\right\} \text { if } r_{i j} \in \Omega_{b}, \min _{1 \leq i \leq m}\left\{r_{i j}\right\} \text { if } r_{i j} \in \Omega_{c}\right), \\
R^{-}= & \left(r_{1}^{-}, r_{2}^{-}, \ldots, r_{n}^{-}\right) \\
& =\left(\min _{1 \leq i \leq m}\left\{r_{i j}\right\} \text { if } r_{i j} \in \Omega_{b}, \max _{1 \leq i \leq m}\left\{r_{i j}\right\} \text { if } r_{i j} \in \Omega_{c}\right),
\end{aligned}
$$

where $\Omega_{b}$ is a set containing benefit-type attributes and $\Omega_{c}$ is a set containing cost-type attributes.

Step 3: calculate the distance between each alterative and PIS as

$$
d_{i}^{+}=\frac{1}{2} \sum_{j=1}^{n} \omega_{j} *\left(\left|r_{i j}-r_{j}^{+}\right|\right), \quad i=1,2, \ldots, m,
$$

and the distance between each alternative and NIS as

$$
d_{i}^{-}=\frac{1}{2} \sum_{j=1}^{n} \omega_{j} *\left(\left|r_{i j}-r_{j}^{-}\right|\right), \quad i=1,2, \ldots, m
$$

Step 4: calculate the relative closeness of each alternative as

$$
\eta_{i}=\frac{d_{i}^{-}}{d_{i}^{+}+d_{i}^{-}}, \quad i=1,2, \ldots, m
$$

Step 5: rank all the alternatives according to their values of relative closeness and select the one with the highest relative closeness as the optimal one.

Step 6: end.

\section{Correlation Coefficient and Entropy Measure}

In this section, the correlation coefficient is developed for LPFSs to measure the correlation degree and two entropy measures are proposed for LPFSs to measure the uncertainty degree.

The distance measure cannot effectively determine the proximity of each alternative to ideal solutions in some special cases. In our study, we plan to replace the distance measure in the TOPSIS method with the correlation coefficient. The correlation coefficient is applied to measure the correlation degree between each alternative and ideal solution.

The attribute weight information in the MADM problems is usually unknown. The entropy measures can reflect the fluctuation of LPFSs in terms of the uncertainty degree. Therefore, the entropy measures can be used to derive the attribute weight information for the MADM problems under the LPFS environment.

3.1. Correlation Coefficients and Their Properties. Suppose that there are a reference set $X=\left\{x_{1}, x_{2}, \ldots, x_{n}\right\}$ and a continuous LTS $S=\left\{s_{\beta} \mid \beta \in[0, \tau]\right\}$, if $A=\left\{\left\langle x_{i}, s_{p_{A}}\left(x_{i}\right)\right.\right.$, $\left.\left.s_{q_{A}}\left(x_{i}\right)\right\rangle \mid x_{i} \in X\right\}$ is an LPFS on $X$, then its information energy is defined as

$$
T(A)=\sum_{i=1}^{n}\left(\left(I\left(s_{p_{A}}\left(x_{i}\right)\right)\right)^{4}+\left(I\left(s_{q_{A}}\left(x_{i}\right)\right)\right)^{4}+\left(I\left(s_{h_{A}}\left(x_{i}\right)\right)\right)^{4}\right),
$$

where $s_{h_{A}}\left(x_{i}\right)$ denotes the hesitance degree of the ith LPFN.

The correlation of between two LPFSs is defined as

$$
\begin{aligned}
C(A, B)= & \sum_{i=1}^{n}\left(\left(I\left(s_{p_{A}}\left(x_{i}\right)\right)\right)^{2}\left(I\left(s_{p_{B}}\left(x_{i}\right)\right)\right)^{2}\right. \\
& +\left(I\left(s_{q_{A}}\left(x_{i}\right)\right)\right)^{2}\left(I\left(s_{q_{B}}\left(x_{i}\right)\right)\right)^{2} \\
& \left.+\left(I\left(s_{h_{A}}\left(x_{i}\right)\right)\right)^{2}\left(I\left(s_{h_{B}}\left(x_{i}\right)\right)\right)^{2}\right) .
\end{aligned}
$$

The correlation of LPFSs has the following properties:

(1) $C(A, A)=T(A)$

(2) $C(A, B)=C(B, A)$

Based on the above information energy and correlation, the correlation coefficient of LPFSs is defined as follows.

Definition 5. Given a finite reference set $X=\left\{x_{1}, x_{2}, \ldots, x_{n}\right\}$ and a continuous LTS $S=\left\{s_{\beta} \mid \beta \in[0, \tau]\right\}$, if $A=\left\{\left\langle x_{i}, s_{p_{A}}\right.\right.$ $\left.\left.\left(x_{i}\right), s_{q_{A}}\left(x_{i}\right)\right\rangle \mid x_{i} \in X\right\}$ and $B=\left\{\left\langle x_{i}, s_{p_{B}}\left(x_{i}\right), s_{q_{B}}\left(x_{i}\right)\right\rangle \mid x_{i} \in\right.$ $X\}$ are two LPFSs on $X$, then the correlation coefficient between them is defined as

$$
\begin{aligned}
K_{1}(A, B) & =\frac{C(A, B)}{[T(A) T(B)]^{1 / 2}} \\
& =\frac{\sum_{i=1}^{n}\left(\left(I\left(s_{p_{A}}\left(x_{i}\right)\right)\right)^{2}\left(I\left(s_{p_{B}}\left(x_{i}\right)\right)\right)^{2}+\left(I\left(s_{q_{A}}\left(x_{i}\right)\right)\right)^{2}\left(I\left(s_{q_{B}}\left(x_{i}\right)\right)\right)^{2}+\left(I\left(s_{h_{A}}\left(x_{i}\right)\right)\right)^{2}\left(I\left(s_{h_{B}}\left(x_{i}\right)\right)\right)^{2}\right)}{\sqrt{\sum_{i=1}^{n}\left(\left(I\left(s_{p_{A}}\left(x_{i}\right)\right)\right)^{4}+\left(I\left(s_{q_{A}}\left(x_{i}\right)\right)\right)^{4}+\left(I\left(s_{h_{A}}\left(x_{i}\right)\right)\right)^{4}\right)} \sqrt{\sum_{i=1}^{n}\left(\left(I\left(s_{p_{B}}\left(x_{i}\right)\right)\right)^{4}+\left(I\left(s_{q_{B}}\left(x_{i}\right)\right)\right)^{4}+\left(I\left(s_{h_{B}}\left(x_{i}\right)\right)\right)^{4}\right)}} .
\end{aligned}
$$

Theorem 1. Given two LPFSs $A=\left\{\left\langle x_{i}, s_{p_{A}}\left(x_{i}\right), s_{q_{A}}\left(x_{i}\right)\right\rangle \mid\right.$ $\left.x_{i} \in X\right\}$ and $B=\left\{\left\langle x_{i}, s_{p_{B}}\left(x_{i}\right), s_{q_{B}}\left(x_{i}\right)\right\rangle \mid x_{i} \in X\right\}$, then the correlation coefficient between them satisfies the following conditions: 
(1) $K_{1}(A, B)=K_{1}(B, A)$

(2) $0 \leq K_{1}(A, B) \leq 1$

(3) $A=B \Longrightarrow K_{1}(A, B)=1$
Proof

(1) It is straightforward.

(2) According to Definition 5, it can be seen that $0 \leq K_{1}$ $(A, B)$. Then, we need to prove that $K_{1}(A, B) \leq 1$ :

$$
\begin{aligned}
C(A, B)= & \sum_{i=1}^{n}\left(\left(I\left(s_{p_{A}}\left(x_{i}\right)\right)\right)^{2}\left(I\left(s_{p_{B}}\left(x_{i}\right)\right)\right)^{2}+\left(I\left(s_{q_{A}}\left(x_{i}\right)\right)\right)^{2}\left(I\left(s_{q_{B}}\left(x_{i}\right)\right)\right)^{2}+\left(I\left(s_{h_{A}}\left(x_{i}\right)\right)\right)^{2}\left(I\left(s_{h_{B}}\left(x_{i}\right)\right)\right)^{2}\right) \\
= & \left(I\left(s_{p_{A}}\left(x_{1}\right)\right)\right)^{2}\left(I\left(s_{p_{B}}\left(x_{1}\right)\right)\right)^{2}+\left(I\left(s_{q_{A}}\left(x_{1}\right)\right)\right)^{2}\left(I\left(s_{q_{B}}\left(x_{1}\right)\right)\right)^{2}+\left(I\left(s_{h_{A}}\left(x_{1}\right)\right)\right)^{2}\left(I\left(s_{h_{B}}\left(x_{1}\right)\right)\right)^{2} \\
& +\left(I\left(s_{p_{A}}\left(x_{2}\right)\right)\right)^{2}\left(I\left(s_{p_{B}}\left(x_{2}\right)\right)\right)^{2}+\left(I\left(s_{q_{A}}\left(x_{2}\right)\right)\right)^{2}\left(I\left(s_{q_{B}}\left(x_{2}\right)\right)\right)^{2}+\left(I\left(s_{h_{A}}\left(x_{2}\right)\right)\right)^{2}\left(I\left(s_{h_{B}}\left(x_{2}\right)\right)\right)^{2} \\
& +\ldots+\left(I\left(s_{p_{A}}\left(x_{n}\right)\right)\right)^{2}\left(I\left(s_{p_{B}}\left(x_{n}\right)\right)\right)^{2}+\left(I\left(s_{q_{A}}\left(x_{n}\right)\right)\right)^{2}\left(I\left(s_{q_{B}}\left(x_{n}\right)\right)\right)^{2}+\left(I\left(s_{h_{A}}\left(x_{n}\right)\right)\right)^{2}\left(I\left(s_{h_{B}}\left(x_{n}\right)\right)\right)^{2} .
\end{aligned}
$$

According to Cauchy-Schwarz inequality $\left(x_{1} y_{1}+\right.$ $\left.x_{2} y_{2}+\ldots+x_{n} y_{n}\right)^{2} \leq\left(x_{1}^{2}+x_{2}^{2}+\ldots+x_{n}^{2}\right) \cdot\left(y_{1}^{2}+y_{2}^{2}+\right.$ $\left.\ldots+y_{n}^{2}\right)$, then we have

$$
\begin{aligned}
(C & (A, B))^{2} \\
\leq & {\left[\left(I\left(s_{p_{A}}\left(x_{1}\right)\right)\right)^{4}+\left(I\left(s_{q_{A}}\left(x_{1}\right)\right)\right)^{4}+\left(I\left(s_{h_{A}}\left(x_{1}\right)\right)\right)^{4}+\left(I\left(s_{p_{A}}\left(x_{2}\right)\right)\right)^{4}+\left(I\left(s_{q_{A}}\left(x_{2}\right)\right)\right)^{4}+\left(I\left(s_{h_{A}}\left(x_{2}\right)\right)\right)^{4}+\ldots+\left(I\left(s_{p_{A}}\left(x_{n}\right)\right)\right)^{4}\right.} \\
& \left.+\left(I\left(s_{q_{A}}\left(x_{n}\right)\right)\right)^{4}+\left(I\left(s_{h_{A}}\left(x_{n}\right)\right)\right)^{4}\right] \\
& \times\left[\left(I\left(s_{p_{B}}\left(x_{1}\right)\right)\right)^{4}+\left(I\left(s_{q_{B}}\left(x_{1}\right)\right)\right)^{4}+\left(I\left(s_{h_{B}}\left(x_{1}\right)\right)\right)^{4}+\left(I\left(s_{p_{B}}\left(x_{2}\right)\right)\right)^{4}+\left(I\left(s_{q_{B}}\left(x_{2}\right)\right)\right)^{4}+\left(I\left(s_{h_{B}}\left(x_{2}\right)\right)\right)^{4}+\ldots+\left(I\left(s_{p_{B}}\left(x_{n}\right)\right)\right)^{4}\right. \\
& \left.+\left(I\left(s_{q_{B}}\left(x_{n}\right)\right)\right)^{4}+\left(I\left(s_{h_{A}}\left(x_{n}\right)\right)\right)^{4}\right] \\
= & \sum_{i=1}^{n}\left(\left(I\left(s_{p_{A}}\left(x_{i}\right)\right)\right)^{4}+\left(I\left(s_{q_{A}}\left(x_{i}\right)\right)\right)^{4}+\left(I\left(s_{h_{A}}\left(x_{i}\right)\right)\right)^{4}\right) \times \sum_{i=1}^{n}\left(\left(I\left(s_{p_{B}}\left(x_{i}\right)\right)\right)^{4}+\left(I\left(s_{q_{B}}\left(x_{i}\right)\right)\right)^{4}+\left(I\left(s_{h_{B}}\left(x_{i}\right)\right)\right)^{4}\right) \\
= & T(A) \cdot T(B) .
\end{aligned}
$$

Thus, $\quad K_{1}(A, B)=\left(C(A, B) /[T(A) T(B)]^{1 / 2}\right) \leq 1$, which completes the proof.

(3) It is straightforward.

In the real applications, the attributes usually have different weights. Thus, the weight of each element $x_{i}$ in the reference set $X$ should be considered when the correlation coefficient is computed. Suppose that $\omega=\left(\omega_{1}, \omega_{2}, \ldots, \omega_{n}\right)$ is the weight vector of the elements in $X$, which satisfies that $\omega_{i} \geq 0$ and $\sum_{i=1}^{n} \omega_{i}=1$, then the weighted correlation coefficient between $A$ and $B$ is defined as

$$
\begin{aligned}
K_{2}(A, B) & =\frac{C_{\omega}(A, B)}{\left[T_{\omega}(A) T_{\omega}(B)\right]^{1 / 2}} \\
& =\frac{\sum_{i=1}^{n} \omega_{i}\left(\left(I\left(s_{p_{A}}\left(x_{i}\right)\right)\right)^{2}\left(I\left(s_{p_{B}}\left(x_{i}\right)\right)\right)^{2}+\left(I\left(s_{q_{A}}\left(x_{i}\right)\right)\right)^{2}\left(I\left(s_{q_{B}}\left(x_{i}\right)\right)\right)^{2}+\left(I\left(s_{h_{A}}\left(x_{i}\right)\right)\right)^{2}\left(I\left(s_{h_{B}}\left(x_{i}\right)\right)\right)^{2}\right)}{\sqrt{\sum_{i=1}^{n} \omega_{i}\left(\left(I\left(s_{p_{A}}\left(x_{i}\right)\right)\right)^{4}+\left(I\left(s_{q_{A}}\left(x_{i}\right)\right)\right)^{4}+\left(I\left(s_{h_{A}}\left(x_{i}\right)\right)\right)^{4}\right)} \sqrt{\sum_{i=1}^{n} \omega_{i}\left(\left(I\left(s_{p_{B}}\left(x_{i}\right)\right)\right)^{4}+\left(I\left(s_{q_{B}}\left(x_{i}\right)\right)\right)^{4}+\left(I\left(s_{h_{B}}\left(x_{i}\right)\right)\right)^{4}\right)}} .
\end{aligned}
$$


Especially, if $\omega=(1 / n, 1 / n, \ldots, 1 / n)$, then $K_{2}(A, B)=$ $K_{1}(A, B)$.

Theorem 2. Given two LPFSs $A=\left\{\left\langle x_{i}, s_{p_{A}}\left(x_{i}\right), s_{q_{A}}\left(x_{i}\right)\right\rangle \mid\right.$ $\left.x_{i} \in X\right\}$ and $B=\left\{\left\langle x_{i}, s_{p_{B}}\left(x_{i}\right), s_{q_{B}}\left(x_{i}\right)\right\rangle \mid x_{i} \in X\right\}$, if $\omega=\left(\omega_{1}\right.$, $\left.\omega_{2}, \ldots, \omega_{n}\right)$ is the weight vector of the elements in $X$, then the weighted correlation coefficient between them satisfies the following conditions:
(1) $K_{2}(A, B)=K_{2}(B, A)$

(2) $0 \leq K_{2}(A, B) \leq 1$

(3) $A=B \Longrightarrow K_{2}(A, B)=1$

Proof

(1) It is straightforward.

(2) It can be noted that $0 \leq K_{2}(A, B)$. We need to prove that $K_{2}(A, B) \leq 1$. According to (4), we have

$$
\begin{aligned}
C_{\omega}(A, B)= & \sum_{i=1}^{n} \omega_{i}\left(\left(I\left(s_{p_{A}}\left(x_{i}\right)\right)\right)^{2}\left(I\left(s_{p_{B}}\left(x_{i}\right)\right)\right)^{2}+\left(I\left(s_{q_{A}}\left(x_{i}\right)\right)\right)^{2}\left(I\left(s_{q_{B}}\left(x_{i}\right)\right)\right)^{2}+\left(I\left(s_{h_{A}}\left(x_{i}\right)\right)\right)^{2}\left(I\left(s_{h_{B}}\left(x_{i}\right)\right)\right)^{2}\right) \\
= & \omega_{1}\left(\left(I\left(s_{p_{A}}\left(x_{1}\right)\right)\right)^{2}\left(I\left(s_{p_{B}}\left(x_{1}\right)\right)\right)^{2}+\left(I\left(s_{q_{A}}\left(x_{1}\right)\right)\right)^{2}\left(I\left(s_{q_{B}}\left(x_{1}\right)\right)\right)^{2}+\left(I\left(s_{h_{A}}\left(x_{1}\right)\right)\right)^{2}\left(I\left(s_{h_{B}}\left(x_{1}\right)\right)\right)^{2}\right) \\
& +\omega_{2}\left(\left(I\left(s_{p_{A}}\left(x_{2}\right)\right)\right)^{2}\left(I\left(s_{p_{B}}\left(x_{2}\right)\right)\right)^{2}+\left(I\left(s_{q_{A}}\left(x_{2}\right)\right)\right)^{2}\left(I\left(s_{q_{B}}\left(x_{2}\right)\right)\right)^{2}+\left(I\left(s_{h_{A}}\left(x_{2}\right)\right)\right)^{2}\left(I\left(s_{h_{B}}\left(x_{2}\right)\right)\right)^{2}\right) \\
& +\ldots+\omega_{n}\left(\left(I\left(s_{p_{A}}\left(x_{n}\right)\right)\right)^{2}\left(I\left(s_{p_{B}}\left(x_{n}\right)\right)\right)^{2}+\left(I\left(s_{q_{A}}\left(x_{n}\right)\right)\right)^{2}\left(I\left(s_{q_{B}}\left(x_{n}\right)\right)\right)^{2}+\left(I\left(s_{h_{A}}\left(x_{n}\right)\right)\right)^{2}\left(I\left(s_{h_{B}}\left(x_{n}\right)\right)\right)^{2}\right) \\
= & \left(\sqrt{\omega_{1}}\left(I\left(s_{p_{A}}\left(x_{1}\right)\right)\right)^{2} \sqrt{\omega_{1}}\left(I\left(s_{p_{B}}\left(x_{1}\right)\right)\right)^{2}+\sqrt{\omega_{1}}\left(I\left(s_{q_{A}}\left(x_{1}\right)\right)\right)^{2} \sqrt{\omega_{1}}\left(I\left(s_{q_{B}}\left(x_{1}\right)\right)\right)^{2}\right. \\
& \left.+\sqrt{\omega_{1}}\left(I\left(s_{h_{A}}\left(x_{1}\right)\right)\right)^{2} \sqrt{\omega_{1}}\left(I\left(s_{h_{B}}\left(x_{1}\right)\right)\right)^{2}\right) \\
& +\left(\sqrt{\omega_{2}}\left(I\left(s_{p_{A}}\left(x_{2}\right)\right)\right)^{2} \sqrt{\omega_{2}}\left(I\left(s_{p_{B}}\left(x_{2}\right)\right)\right)^{2}+\sqrt{\omega_{2}}\left(I\left(s_{q_{A}}\left(x_{2}\right)\right)\right)^{2} \sqrt{\omega_{2}}\left(I\left(s_{q_{B}}\left(x_{2}\right)\right)\right)^{2}\right. \\
& \left.+\sqrt{\omega_{2}}\left(I\left(s_{h_{A}}\left(x_{2}\right)\right)\right)^{2} \sqrt{\omega_{2}}\left(I\left(s_{h_{B}}\left(x_{2}\right)\right)\right)^{2}\right)+\ldots \\
& +\left(\sqrt{\omega_{n}}\left(I\left(s_{p_{A}}\left(x_{n}\right)\right)\right)^{2} \sqrt{\omega_{n}}\left(I\left(s_{p_{B}}\left(x_{n}\right)\right)\right)^{2}+\sqrt{\omega_{n}}\left(I\left(s_{q_{A}}\left(x_{n}\right)\right)\right)^{2} \sqrt{\omega_{n}}\left(I\left(s_{q_{B}}\left(x_{n}\right)\right)\right)^{2}\right. \\
& \left.+\sqrt{\omega_{n}}\left(I\left(s_{h_{A}}\left(x_{n}\right)\right)\right)^{2} \sqrt{\omega_{n}}\left(I\left(s_{h_{B}}\left(x_{n}\right)\right)\right)^{2}\right) .
\end{aligned}
$$

According to Cauchy-Schwarz inequality, we have

$$
\begin{aligned}
\left(C_{\omega}(A, B)\right)^{2} \leq & {\left[\omega_{1}\left(\left(I\left(s_{p_{A}}\left(x_{1}\right)\right)\right)^{4}+\left(I\left(s_{q_{A}}\left(x_{1}\right)\right)\right)^{4}+\left(I\left(s_{h_{A}}\left(x_{1}\right)\right)\right)^{4}\right)+\omega_{2}\left(\left(I\left(s_{p_{A}}\left(x_{2}\right)\right)\right)^{4}+\left(I\left(s_{q_{A}}\left(x_{2}\right)\right)\right)^{4}+\left(I\left(s_{h_{A}}\left(x_{2}\right)\right)\right)^{4}\right)\right.} \\
& \left.+\ldots+\omega_{n}\left(\left(I\left(s_{p_{A}}\left(x_{n}\right)\right)\right)^{4}+\left(I\left(s_{q_{A}}\left(x_{n}\right)\right)\right)^{4}+\left(I\left(s_{h_{A}}\left(x_{n}\right)\right)\right)^{4}\right)\right] \times\left[\omega_{1}\left(\left(I\left(s_{p_{B}}\left(x_{1}\right)\right)\right)^{4}+\left(I\left(s_{q_{B}}\left(x_{1}\right)\right)\right)^{4}+\left(I\left(s_{h_{B}}\left(x_{1}\right)\right)\right)^{4}\right)\right. \\
& \left.+\omega_{2}\left(\left(I\left(s_{p_{B}}\left(x_{2}\right)\right)\right)^{4}+\left(I\left(s_{q_{B}}\left(x_{2}\right)\right)\right)^{4}+\left(I\left(s_{h_{B}}\left(x_{2}\right)\right)\right)^{4}\right)+\ldots+\omega_{n}\left(\left(I\left(s_{p_{B}}\left(x_{n}\right)\right)\right)^{4}+\left(I\left(s_{q_{B}}\left(x_{n}\right)\right)\right)^{4}+\left(I\left(s_{h_{B}}\left(x_{n}\right)\right)\right)^{4}\right)\right] \\
= & \sum_{i=1}^{n} \omega_{i}\left(\left(I\left(s_{p_{A}}\left(x_{i}\right)\right)\right)^{4}+\left(I\left(s_{q_{A}}\left(x_{i}\right)\right)\right)^{4}+\left(I\left(s_{h_{A}}\left(x_{i}\right)\right)\right)^{4}\right) \times \sum_{i=1}^{n} \omega_{i}\left(\left(I\left(s_{p_{B}}\left(x_{i}\right)\right)\right)^{4}+\left(I\left(s_{q_{B}}\left(x_{i}\right)\right)\right)^{4}+\left(I\left(s_{h_{B}}\left(x_{i}\right)\right)\right)^{4}\right) \\
= & T_{\omega}(A) \cdot T_{\omega}(B) .
\end{aligned}
$$

Then, we have $K_{2}(A, B)=\left(C_{\omega}(A, B) /\left[T_{\omega}(A) T_{\omega}\right.\right.$ $\left.(B)]^{1 / 2}\right) \leq 1$, which completes the proof.

(3) It is straightforward.
3.2. Entropy Measures and Their Properties. For the MADM problems, the weights of attributes are usually unknown due to the time pressure, the DMs' limited capability, and the 
complexity of the considered problems. The entropy measure is an efficient tool to derive the weights of attributes [56]. Ye [57] devised two entropy measures to determine the weights of attributes in the MADM problems with intervalvalued IFSs. Xia and $\mathrm{Xu}$ [58] put forward the entropy and cross-entropy measures for IFSs, based on which, the weights of attributes are computed. Xu and Xia [59] put forward the entropy and cross entropy for hesitant fuzzy sets and used the entropy measure to determine the weight vector of attributes. Nevertheless, the entropy measure of LPFSs has not been proposed. To measure the uncertainty degree of LPFSs, we propose two entropy measures for LPFSs and discuss their properties.

Definition 6. Given a reference set $X=\left\{x_{1}, x_{2}, \ldots, x_{n}\right\}$, a continuous LTS $S=\left\{s_{\beta} \mid \beta \in[0, \tau]\right\}$, and an LPFS $A=\left\{\left\langle x_{i}\right.\right.$, $\left.\left.s_{p_{A}}\left(x_{i}\right), s_{q_{A}}\left(x_{i}\right)\right\rangle \mid x_{i} \in X\right\}$, two entropy measures for the LPFS are defined as

$$
\begin{aligned}
& E_{1}(A)=\frac{1}{n} \sum_{i=1}^{n}\left\{\left\{\sin \frac{\pi \times\left[\tau^{2}+\left(I\left(s_{p_{A}}\left(x_{i}\right)\right)\right)^{2}-\left(I\left(s_{q_{A}}\left(x_{i}\right)\right)\right)^{2}\right]}{4 \tau^{2}}+\sin \frac{\pi \times\left[\tau^{2}-\left(I\left(s_{p_{A}}\left(x_{i}\right)\right)\right)^{2}+\left(I\left(s_{q_{A}}\left(x_{i}\right)\right)\right)^{2}\right]}{4 \tau^{2}}-1\right\} \times \frac{1}{\sqrt{2}-1}\right\}, \\
& E_{2}(A)=\frac{1}{n} \sum_{i=1}^{n}\left\{\left\{\cos \frac{\pi \times\left[\tau^{2}+\left(I\left(s_{p_{A}}\left(x_{i}\right)\right)\right)^{2}-\left(I\left(s_{q_{A}}\left(x_{i}\right)\right)\right)^{2}\right]}{4 \tau^{2}}+\cos \frac{\pi \times\left[\tau^{2}-\left(I\left(s_{p_{A}}\left(x_{i}\right)\right)\right)^{2}+\left(I\left(s_{q_{A}}\left(x_{i}\right)\right)\right)^{2}\right]}{4 \tau^{2}}-1\right\} \times \frac{1}{\sqrt{2}-1}\right\},
\end{aligned}
$$

where $I\left(s_{p_{A}}\left(x_{i}\right)\right)$ and $I\left(s_{q_{A}}\left(x_{i}\right)\right)$ denote the subscripts of the MD and NMD of the ith element $x_{i}$.

Theorem 3. Given two LPFSs $A=\left\{\left\langle x_{i}, s_{p_{A}}\left(x_{i}\right), s_{q_{A}}\left(x_{i}\right)\right\rangle \mid\right.$ $\left.x_{i} \in X\right\}$ and $B=\left\{\left\langle x_{i}, s_{p_{B}}\left(x_{i}\right), s_{q_{B}}\left(x_{i}\right)\right\rangle \mid x_{i} \in X\right\}$, the entropy measures of them satisfy the following properties:

(1) If $s_{p_{A}}\left(x_{i}\right)=s_{0}, s_{q_{A}}\left(x_{i}\right)=s_{\tau}$ or $s_{p_{A}}\left(x_{i}\right)=s_{\tau}, s_{q_{A}}\left(x_{i}\right)=$ $s_{0}$, then $E_{1}(A)=E_{2}(A)=0$

(2) If $s_{p_{A}}\left(x_{i}\right)=s_{q_{A}}\left(x_{i}\right)$, then $E_{1}(A)=E_{2}(A)=1$
(3) $E_{1}(A) \leq E_{1}(B)$ and $E_{2}(A) \leq E_{2}(B)$ if $A$ is less fuzzy than $B$, namely, $s_{p_{A}}\left(x_{i}\right) \leq s_{p_{B}}\left(x_{i}\right)$ and $s_{q_{A}}\left(x_{i}\right) \geq$ $s_{q_{B}}\left(x_{i}\right)$ for $s_{p_{B}}\left(x_{i}\right) \leq s_{q_{B}}\left(x_{i}\right)$ or $s_{p_{A}}\left(x_{i}\right) \geq s_{p_{B}}\left(x_{i}\right)$ and $s_{q_{A}}\left(x_{i}\right) \leq s_{q_{B}}\left(x_{i}\right)$ for $s_{p_{B}}\left(x_{i}\right) \geq s_{q_{B}}\left(x_{i}\right)$

(4) $E_{1}(A)=E_{1}\left(A^{c}\right)$ and $E_{2}(A)=E_{2}\left(A^{c}\right)$

Proof

(1) To make the proof process be easy, let

$$
\begin{aligned}
& E_{1}\left(A\left(x_{i}\right)\right)=\left\{\sin \frac{\pi \times\left[\tau^{2}+\left(I\left(s_{p_{A}}\left(x_{i}\right)\right)\right)^{2}-\left(I\left(s_{q_{A}}\left(x_{i}\right)\right)\right)^{2}\right]}{4 \tau^{2}}+\sin \frac{\pi \times\left[\tau^{2}-\left(I\left(s_{p_{A}}\left(x_{i}\right)\right)\right)^{2}+\left(I\left(s_{q_{A}}\left(x_{i}\right)\right)\right)^{2}\right]}{4 \tau^{2}}-1\right\} \times \frac{1}{\sqrt{2}-1}, \\
& E_{2}\left(A\left(x_{i}\right)\right)=\left\{\cos \frac{\pi \times\left[\tau^{2}+\left(I\left(s_{p_{A}}\left(x_{i}\right)\right)\right)^{2}-\left(I\left(s_{q_{A}}\left(x_{i}\right)\right)\right)^{2}\right]}{4 \tau^{2}}+\cos \frac{\pi \times\left[t^{2}-\left(I\left(s_{p_{A}}\left(x_{i}\right)\right)\right)^{2}+\left(I\left(s_{q_{A}}\left(x_{i}\right)\right)\right)^{2}\right]}{4 \tau^{2}}-1\right\} \times \frac{1}{\sqrt{2}-1} .
\end{aligned}
$$


If $s_{p_{A}}\left(x_{i}\right)=s_{0}, s_{q_{A}}\left(x_{i}\right)=s_{\tau}$, or $s_{p_{A}}\left(x_{i}\right)=s_{\tau}, s_{q_{A}}\left(x_{i}\right)=$ $s_{0}$, then $E_{1}\left(A\left(x_{i}\right)\right)=E_{2}\left(A\left(x_{i}\right)\right)=0$. Hence, we can get $E_{1}(A)=E_{2}(A)=0$.

(2) If $s_{p_{A}}\left(x_{i}\right)=s_{q_{A}}\left(x_{i}\right)$, then we have $E_{1}\left(A\left(x_{i}\right)\right)=E_{2}$ $\left(A\left(x_{i}\right)\right)=1$. Thus, $E_{1}(A)=E_{2}(A)=1$.

(3) Let $x=I\left(s_{p_{A}}\left(x_{i}\right)\right)$ and $y=I\left(s_{q_{A}}\left(x_{i}\right)\right)$, then equations (19) and (20) can be transformed as

$$
\begin{aligned}
F_{1}(x, y)= & \left\{\sin \frac{\pi \times\left[\tau^{2}+x^{2}-y^{2}\right]}{4 \tau^{2}}\right. \\
& \left.+\sin \frac{\pi \times\left[\tau^{2}-x^{2}+y^{2}\right]}{4 \tau^{2}}-1\right\} \times \frac{1}{\sqrt{2}-1}, \\
F_{2}(x, y)= & \left\{\cos \frac{\pi \times\left[\tau^{2}+x^{2}-y^{2}\right]}{4 \tau^{2}}\right. \\
& \left.+\cos \frac{\pi \times\left[\tau^{2}-x^{2}+y^{2}\right]}{4 \tau^{2}}-1\right\} \times \frac{1}{\sqrt{2}-1} .
\end{aligned}
$$

Thus, to prove that equations (17) and (18) satisfy the property (11), we need to prove that the functions $F_{1}(x, y)$ and $F_{2}(x, y)$ are increasing with respect to its parameter $x$ and decreasing for the parameter $y$. Taking the partial derivative of the functions $F_{1}(x, y)$ and $F_{2}(x, y)$ with respect to $x$ and $y$ yields

$$
\begin{aligned}
\frac{\partial F_{1}(x, y)}{\partial x}= & \frac{\pi \times 2 x}{4 \tau^{2}(\sqrt{2}-1)}\left[\cos \frac{\pi \times\left[\tau^{2}+x^{2}-y^{2}\right]}{4 \tau^{2}}\right. \\
& \left.-\cos \frac{\pi \times\left[\tau^{2}-x^{2}+y^{2}\right]}{4 \tau^{2}}\right], \\
\frac{\partial F_{1}(x, y)}{\partial y}= & \frac{\pi \times 2 y}{4 \tau^{2}(\sqrt{2}-1)}\left[\cos \frac{\pi \times\left[\tau^{2}-x^{2}+y^{2}\right]}{4 \tau^{2}}\right. \\
& \left.-\cos \frac{\pi \times\left[\tau^{2}+x^{2}-y^{2}\right]}{4 \tau^{2}}\right], \\
\frac{\partial F_{2}(x, y)}{\partial x}= & \frac{\pi \times 2 x}{4 \tau^{2}(\sqrt{2}-1)}\left[\sin \frac{\pi \times\left[\tau^{2}-x^{2}+y^{2}\right]}{4 \tau^{2}}\right. \\
& \left.-\sin \frac{\pi \times\left[\tau^{2}+x^{2}-y^{2}\right]}{4 \tau^{2}}\right], \\
\frac{\partial F_{2}(x, y)}{\partial y}= & \frac{\pi \times 2 y}{4 \tau^{2}(\sqrt{2}-1)}\left[\sin \frac{\pi \times\left[\tau^{2}+x^{2}-y^{2}\right]}{4 \tau^{2}}\right. \\
& \left.-\sin \frac{\pi \times\left[\tau^{2}-x^{2}+y^{2}\right]}{4 \tau^{2}}\right] .
\end{aligned}
$$

To obtain the critical point of the functions $F_{1}(x, y)$ and $F_{2}(x, y)$, let $\partial F_{1}(x, y) / \partial x=0, \quad \partial F_{1}(x, y) / \partial y=0$, $\partial F_{2}(x, y) / \partial x=0$, and $\partial F_{2}(x, y) / \partial y=0$. By solving these four equations, the critical point can be obtained as

$$
x_{c p}=y \text {. }
$$

From equations (22), (24), and (26), we have

$$
\begin{aligned}
& \frac{\partial F_{1}(x, y)}{\partial x} \geq 0 \text { for } x \leq y \text { and } \frac{\partial F_{1}(x, y)}{\partial x} \leq 0 \text { for } x \geq y, \\
& \frac{\partial F_{2}(x, y)}{\partial x} \geq 0 \text { for } x \leq y \text { and } \frac{\partial F_{2}(x, y)}{\partial x} \leq 0 \text { for } x \geq y .
\end{aligned}
$$

Thus, it can be noted that the functions $F_{1}(x, y)$ and $F_{2}(x, y)$ are increasing with respect to $x$ for $x \leq y$ and decreasing for $x \geq y$. Similarly, it can be derived that

$$
\begin{aligned}
& \frac{\partial F_{1}(x, y)}{\partial y} \leq 0 \text { for } x \leq y \text { and } \frac{\partial F_{1}(x, y)}{\partial y} \geq 0 \text { for } x \geq y, \\
& \frac{\partial F_{2}(x, y)}{\partial y} \leq 0 \text { for } x \leq y \text { and } \frac{\partial F_{2}(x, y)}{\partial y} \geq 0 \text { for } x \geq y .
\end{aligned}
$$

Then, it can be seen that the functions $F_{1}(x, y)$ and $F_{2}(x, y)$ are decreasing with respect to $y$ for $x \leq y$ and increasing for $x \geq y$.

Let us now consider equations (19) and (20) with $A \leq B$. Assume that the reference set $X$ is divided into two parts $X_{1}$ and $X_{2}$ satisfying $X_{1} \cup X_{2}=X$. When $s_{p_{A}}\left(x_{i}\right) \leq s_{p_{B}}\left(x_{i}\right) \leq s_{q_{B}}\left(x_{i}\right) \leq s_{q_{A}}\left(x_{i}\right)$ for $x_{i} \in X_{1}$, or $s_{p_{A}}\left(x_{i}\right) \geq s_{p_{B}}\left(x_{i}\right) \geq s_{q_{B}}\left(x_{i}\right) \geq s_{q_{A}}\left(x_{i}\right)$ for $x_{i} \in X_{2}$, then we can achieve that $E_{1}\left(A\left(x_{i}\right)\right) \leq E_{1}\left(B\left(x_{i}\right)\right)$ and $E_{2}\left(A\left(x_{i}\right)\right) \leq E_{2}\left(B\left(x_{i}\right)\right)$ according to the monotonicity of the functions $F_{1}(x, y)$ and $F_{2}(x, y)$.

(4) Since $A^{c}=\left\{\left\langle x_{i}, s_{q_{A}}\left(x_{i}\right), s_{p_{A}}\left(x_{i}\right)\right\rangle \mid x \in X\right\}$ for $x_{i} \in X_{1}$, i.e., $s_{p_{A^{c}}}\left(x_{i}\right)=s_{q_{A}}\left(x_{i}\right)$ and $s_{q_{A c}}\left(x_{i}\right)=s_{p_{A}}\left(x_{i}\right)$, then we have $E_{1}\left(A\left(x_{i}\right)\right)=E_{1}\left(A^{c}\left(x_{i}\right)\right)$ and $E_{2}\left(A\left(x_{i}\right)\right)=E_{2}$ $\left(A^{c}\left(x_{i}\right)\right)$ according to equations (19) and (20). Hence, $E_{1}(A)=E_{1}\left(A^{c}\right)$ and $E_{2}(A)=E_{2}\left(A^{c}\right)$.

\section{Novel TOPSIS Method Based on Correlation Coefficient and Entropy Measure}

In this section, a novel TOPSIS method based on correlation coefficient and entropy measure is given to handle the MADM problems under the LPFS environment.

4.1. Problem Description. Consider an MADM problem that is composed of $m$ alternatives denoted as $C=\left\{c_{1}, c_{2}, \ldots, c_{m}\right\}$ and $n$ attributes denoted as $U=\left\{u_{1}, u_{2}, \ldots, u_{n}\right\}$. The weights of attributes are expressed as $\omega=\left(\omega_{1}, \omega_{2}, \ldots, \omega_{n}\right)$, which satisfy $\omega_{j} \geq 0$ and $\sum_{j=1}^{n} \omega_{j}=1$. Each attribute of each alternative is evaluated using an LPFN: 


$$
r_{i j}=\left(s_{p_{i j}}, s_{q_{i j}}\right),
$$

where $s_{p_{i j}}$ and $s_{q_{i j}}$ are the elements in a continuous LTS $S=\left\{s_{\beta} \mid \beta \in[0, \tau]\right\}$, which satisfy the condition $p_{i j}{ }^{2}+q_{i j}{ }^{2} \leq \tau^{2}$. All the attribute values of the alternatives can form a linguistic Pythagorean fuzzy decision matrix (LPFDM) $R=\left(r_{i j}\right)_{m \times n}=\left(\left(S_{p_{i j}}, S_{q_{i j}}\right)\right)_{m \times n} \quad$ where $\quad i=1,2, \ldots, m$, $j=1,2, \ldots, n$.
Because of the time pressure, limited professional knowledge of DMs, and complexity of problems, it is difficult to provide the weight information of attributes in advance. To derive the weights of attributes, we use the proposed entropy measures to compute the weight of each attribute as

$$
\omega_{j}=\frac{1-H_{j}}{n-\sum_{j=1}^{n} H_{j}},
$$

where $\omega_{j} \in[0,1], \sum_{j=1}^{n} \omega_{j}=1$, and $H_{j}$ is calculated as

$$
\begin{array}{r}
H_{j}=\frac{1}{m} \sum_{i=1}^{m}\left\{\left\{\sin \frac{\pi \times\left[\tau^{2}+\left(I\left(s_{p_{i j}}\right)\right)^{2}-\left(I\left(s_{q_{i j}}\right)\right)^{2}\right]}{4 \tau^{2}}+\sin \frac{\pi \times\left[\tau^{2}-\left(I\left(s_{p_{i j}}\right)\right)^{2}+\left(I\left(s_{q_{i j}}\right)\right)^{2}\right]}{4 \tau^{2}}-1\right\} \times \frac{1}{\sqrt{2}-1}\right\} \\
\text { or } H_{j}=\frac{1}{m} \sum_{i=1}^{m}\left\{\left\{\cos \frac{\pi \times\left[\tau^{2}+\left(I\left(s_{p_{i j}}\right)\right)^{2}-\left(I\left(s_{q_{i j}}\right)\right)^{2}\right]}{4 \tau^{2}}+\cos \frac{\pi \times\left[\tau^{2}-\left(I\left(s_{p_{i j}}\right)\right)^{2}+\left(I\left(s_{q_{i j}}\right)\right)^{2}\right]}{4 \tau^{2}}-1\right\} \times \frac{1}{\sqrt{2}-1}\right\},
\end{array}
$$

and $H_{j} \in[0,1]$ for $j=1,2, \ldots, n$.

4.2. The Decision-Making Procedure. According to the above analysis, the decision-making procedure of our proposed LPF-TOPSIS based on correlation coefficient and entropy measure is summarized as follows:

Step 1: in the MADM problems, there are benefit-type and cost-type attributes. Therefore, the LPFDM $R=$ $\left(r_{i j}\right)_{m \times n}$ should be normalized as $\bar{R}=\left(\bar{r}_{i j}\right)_{m \times n}$, where

$$
\bar{r}_{i j}=\left(\bar{s}_{p_{i j}}, \bar{s}_{q_{i j}}\right)=\left\{\begin{array}{l}
\left(s_{p_{i j}}, s_{q_{i j}}\right) \text { for benefit }- \text { type attribute } u_{j}, \\
\left(s_{q_{i j}}, s_{p_{i j}}\right) \text { for cost }- \text { type attribute } u_{j} .
\end{array}\right.
$$

Step 2: use equation (30) to determine the weight vector of attributes.

Step 3: according to the LPFDM $\bar{R}=\left(\bar{r}_{i j}\right)_{m \times n}$, the PIS and NIS among the alternatives are determined as

$$
\begin{aligned}
& \bar{R}^{+}=\left(\bar{r}_{1}^{+}, \bar{r}_{2}^{+}, \ldots, \bar{r}_{n}^{+}\right), \\
& \bar{R}^{-}=\left(\bar{r}_{1}^{-}, \bar{r}_{2}^{-}, \ldots, \bar{r}_{n}^{-}\right) .
\end{aligned}
$$

where $\bar{r}_{j}^{+}=\left(\bar{s}_{p_{\max } j}, \bar{s}_{q_{\min j}}\right)=\left(\max _{1 \leq i \leq m} \bar{s}_{p_{i j}}, \min _{1 \leq i \leq m} \bar{s}_{q_{i j}}\right)$ and $\bar{r}_{j}^{-}=\left(\bar{s}_{p_{\min } j} \bar{s}_{q_{\max }}\right)=\left(\min _{1 \leq i \leq m} \bar{s}_{p_{i j}}, \max _{1 \leq i \leq m} \bar{s}_{q_{i j}}\right)$.

Step 4: according to equation (14), the weighted correlation coefficient value between each alternative and linguistic Pythagorean fuzzy PIS is computed as

$$
K_{i}^{+}\left(\bar{r}_{i j}, \bar{R}^{+}\right)=\frac{\sum_{j=1}^{n} \omega_{j}\left(\left(I\left(\bar{s}_{p_{i j}}\right)\right)^{2}\left(I\left(\bar{s}_{p_{\max }}\right)\right)^{2}+\left(I\left(\bar{s}_{q_{i j}}\right)\right)^{2}\left(I\left(\bar{s}_{q_{\min j}}\right)\right)^{2}+\left(I\left(\bar{s}_{h_{i j}}\right)\right)^{2}\left(I\left(\bar{s}_{h_{\max } \min j}\right)\right)^{2}\right)}{\sqrt{\sum_{j=1}^{n} \omega_{j}\left(\left(I\left(\bar{s}_{p_{i j}}\right)\right)^{4}+\left(I\left(\bar{s}_{q_{i j}}\right)\right)^{4}+\left(I\left(\bar{s}_{h_{i j}}\right)\right)^{4}\right)} \sqrt{\sum_{j=1}^{n} \omega_{j}\left(\left(I\left(\bar{s}_{p_{\max }}\right)\right)^{4}+\left(I\left(\bar{s}_{q_{\min j}}\right)\right)^{4}+\left(I\left(\bar{s}_{h_{\max \min j}}\right)\right)^{4}\right)}},
$$


and the weighted correlation coefficient between each alternative and the linguistic Pythagorean fuzzy NIS is computed as

$$
K_{i}^{-}\left(\bar{r}_{i j}, \bar{R}^{-}\right)=\frac{\sum_{j=1}^{n} \omega_{j}\left(\left(I\left(\bar{s}_{p_{i j}}\right)\right)^{2}\left(I\left(\bar{s}_{p_{\min j}}\right)\right)^{2}+\left(I\left(\bar{s}_{q_{i j}}\right)\right)^{2}\left(I\left(\bar{s}_{q_{\max j}}\right)\right)^{2}+\left(I\left(\bar{s}_{h_{i j}}\right)\right)^{2}\left(I\left(\bar{s}_{h_{\min m a x}}\right)\right)^{2}\right)}{\sqrt{\sum_{j=1}^{n} \omega_{j}\left(\left(I\left(\bar{s}_{p_{i j}}\right)\right)^{4}+\left(I\left(\bar{s}_{q_{i j}}\right)\right)^{4}+\left(I\left(\bar{s}_{h_{i j}}\right)\right)^{4}\right)} \sqrt{\sum_{j=1}^{n} \omega_{j}\left(\left(I\left(\bar{s}_{p_{\min j}}\right)\right)^{4}+\left(I\left(\bar{s}_{q_{\max }}\right)\right)^{4}+\left(I\left(\bar{s}_{h_{\min } \max j}\right)\right)^{4}\right)}}
$$

where $I\left(\bar{s}_{h_{\max \min j}}\right)$ is the HD of the LPFN $\bar{r}_{j}^{+}$and $I\left(\bar{s}_{h_{\min \max }}\right)$ is the HD of the LPFN $\bar{r}_{j}^{-}$.

Step 5: based on the weighted correlation coefficients, the relative closeness of each alternative can be calculated as

$$
\eta_{i}=\frac{K_{i}^{+}\left(\bar{r}_{i j}, \bar{R}^{+}\right)}{K_{i}^{+}\left(\bar{r}_{i j}, \bar{R}^{+}\right)+K_{i}^{-}\left(\bar{r}_{i j}, \bar{R}^{-}\right)} .
$$

Step 6: all the alternatives are ranked according to their values of relative closeness and the alternative with the highest value of relative closeness is selected as the optimal one.

Step 7: end.

The decision-making procedure of the proposed LPFTOPSIS method based on correlation coefficient and entropy measure is depicted as shown in Figure 2. It can be noted that the proposed LPF-TOPSIS method can solve the MADM problems with unknown attribute weight information using entropy measures. It uses the weighted correlation coefficient to replace the distance measure so as to improve the decision results of the TOPSIS method. Therefore, our study can not only enrich the theoretical basis related to the LPFSs but also promote the wide applications of LPFSs in various fields.

\section{Case Study and Comparative Analyses}

In this section, a practical problem concerning the selection of firewall productions is provided to show the implementation process of the proposed LPF-TOPSIS method and the security evaluation of computer systems is given to compare the proposed LPF-TOPSIS method with the previous decision-making methods.

5.1. Case Study: The Selection of Firewall Productions. The firewall refers to a security gateway that protects the computer networks from malicious intrusions [60-62]. There are many kinds of firewall productions in the market, which have different advantages and disadvantages. Hence, how to select an appropriate firewall production according to their factors is a typical MADM problem.
Example 1. The intranet of an enterprise is usually attacked by malicious intrusions. To enhance the security of the intranet, this enterprise plans to purchase the firewall production and deploys it between the intranet and extranet for blocking illegal accesses. Currently, there are five kinds of firewall productions available to be chosen, which are denoted as $C=\left\{c_{1}, c_{2}, c_{3}, c_{4}, c_{5}\right\}$. When selecting the firewall production, the enterprise pays attention to the factors, which are the promotion $\left(u_{1}\right)$, configuration simplicity $\left(u_{2}\right)$, security level $\left(u_{3}\right)$, and maintenance service level $\left(u_{4}\right)$. The weights of these attributes are $\omega=\left(\omega_{1}, \omega_{2}, \omega_{3}, \omega_{4}\right)$, which satisfy that $\omega_{j} \geq 0$ and $\sum_{j=1}^{4} \omega_{j}=1$. To evaluate the firewall productions with respect to their factors, the enterprise chooses a pair of linguistic terms from the following LTS:

$$
\begin{aligned}
S=\left\{s_{0}\right. & =\text { extremely poor, } s_{1}=\text { very poor, } s_{2}=\text { poor }, \\
s_{3} & =\text { slightly poor, } s_{4}=\text { fair, } s_{5}=\text { slightly good, } \\
s_{6} & \left.=\text { good, } s_{7}=\text { very good, } s_{8}=\text { extremely good }\right\},
\end{aligned}
$$

to express their evaluation information as LPFNs. All the LPFNs form an LPFDM $R=\left(r_{i j}\right)_{5 \times 4}$ as shown in Table 1 .

People expect to select then productions having low price but high quality. There is no exception to the firewall productions. For all the attributes, the higher the attribute values are, the better the alternatives are. Hence, all the attributes are benefit-type.

Step 1: since all the attributes are benefit-type, it is unnecessary to normalize the LPFDM $R=\left(r_{i j}\right)_{5 \times 4}$.

Step 2: using equation (30), the weights of the attributes can be computed as

$$
\omega=(0.2190,0.3229,0.2636,0.1945) \text {. }
$$

Step 3: according to the LPFDM, the linguistic Pythagorean fuzzy PIS and NIS can be obtained as

$$
\begin{aligned}
& R^{+}=\left[\left(s_{7}, s_{1}\right),\left(s_{7}, s_{1}\right),\left(s_{7}, s_{1}\right),\left(s_{6}, s_{1}\right)\right], \\
& R^{-}=\left[\left(s_{4}, s_{3}\right),\left(s_{5}, s_{3}\right),\left(s_{5}, s_{2}\right),\left(s_{5}, s_{4}\right)\right] .
\end{aligned}
$$

Step 4: using equation (34), the weighted correlation coefficient between each alternative and the linguistic Pythagorean fuzzy PIS is computed as 


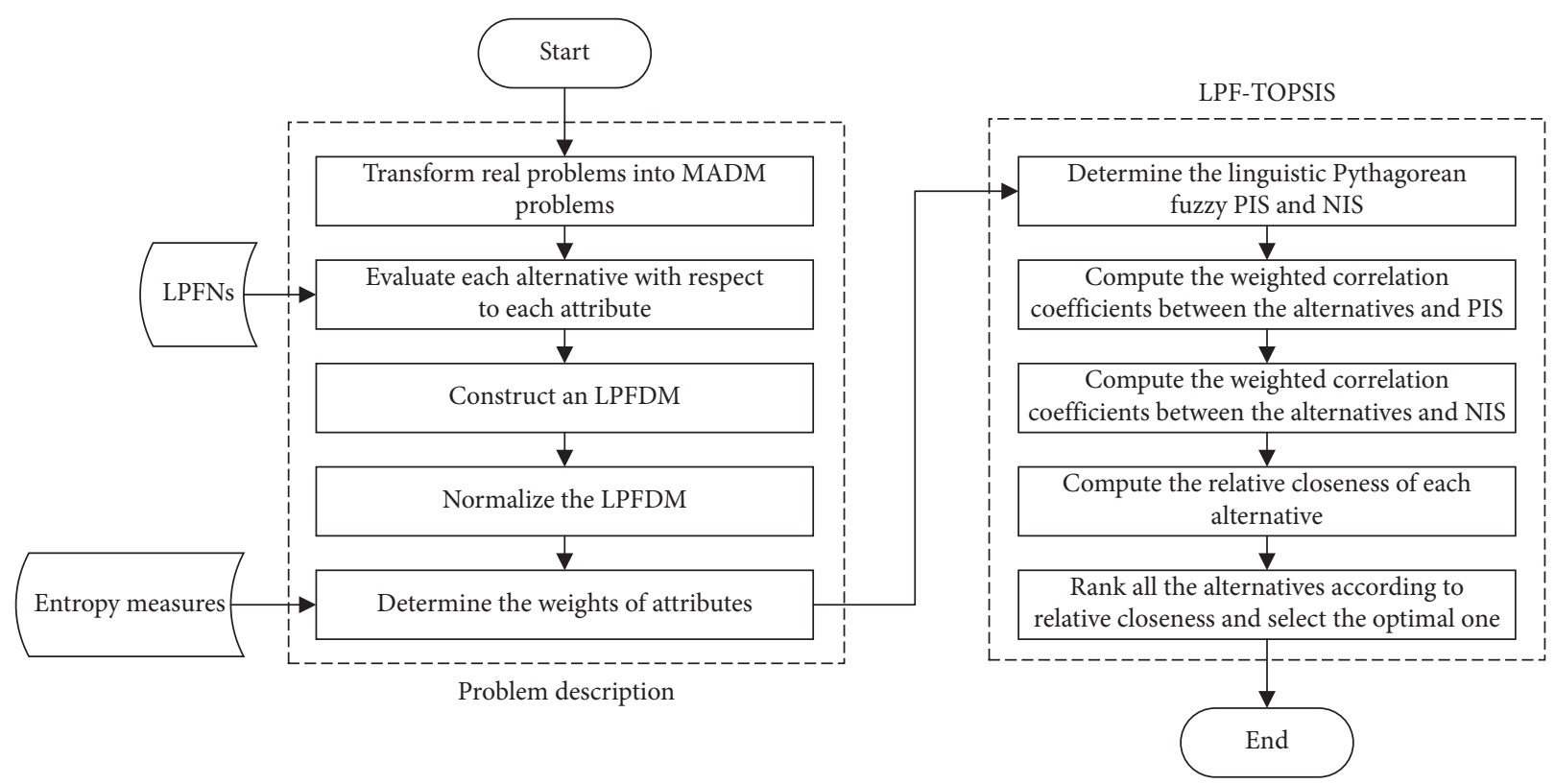

FIGURE 2: The decision-making procedure of the LPF-TOPSIS method.

$$
\begin{aligned}
& K_{1}^{+}\left(r_{1 j}, R^{+}\right)=0.8028, \\
& K_{2}^{+}\left(r_{2 j}, R^{+}\right)=0.7721, \\
& K_{3}^{+}\left(r_{3 j}, R^{+}\right)=0.8513, \\
& K_{4}^{+}\left(r_{4 j}, R^{+}\right)=0.8058, \\
& K_{5}^{+}\left(r_{5 j}, R^{+}\right)=0.9248 .
\end{aligned}
$$

Using equation (35), the weighted correlation coefficient between each alternative and the linguistic Pythagorean fuzzy NIS is computed as

$$
\begin{aligned}
& K_{1}^{-}\left(r_{1 j}, R^{-}\right)=0.8657, \\
& K_{2}^{-}\left(r_{2 j}, R^{-}\right)=0.9246, \\
& K_{3}^{-}\left(r_{3 j}, R^{-}\right)=0.9068, \\
& K_{4}^{-}\left(r_{4 j}, R^{-}\right)=0.8818, \\
& K_{5}^{-}\left(r_{5 j}, R^{-}\right)=0.7695 .
\end{aligned}
$$

Step 5: using equation (36), the relative closeness of each alternative is calculated as

$$
\begin{aligned}
& \eta_{1}=0.4812, \\
& \eta_{2}=0.4551, \\
& \eta_{3}=0.4842, \\
& \eta_{4}=0.4775, \\
& \eta_{5}=0.5458 .
\end{aligned}
$$

Step 6: according to the values of relative closeness, all the alternatives can be ranked as

$$
c_{5}>c_{3}>c_{1}>c_{4}>c_{2} .
$$

Then, the optimal one is $c_{5}$.

Step 7: end.

5.2. Comparative Analysis. In this subsection, an example concerning the security evaluation of computer systems is provided to compare the proposed LPF-TOPSIS method with some decision-making methods, which are the proposed TOPSIS method based on distance measure and the existing decision-making methods based on aggregation operators $[41,42]$.

Example 2. The Information Technology Security Evaluation Center is an authoritative organization, which provides security evaluation services for companies. They are authorized to evaluate four computer systems $C=\left\{c_{1}, c_{2}, c_{3}, c_{4}\right\}$ according to their factors, which are the hardware security $\left(u_{1}\right)$, system software security $\left(u_{2}\right)$, application software security $\left(u_{3}\right)$, and data security $\left(u_{4}\right)$. For these four factors, the higher the values are, the better the alternatives are. Hence, all of these attributes are benefittype. Each alternative concerning each attribute is evaluated using the LPFNs, whose MD and NMD are from the following LTS:

$$
\begin{aligned}
S=\left\{s_{0}\right. & =\text { extremely poor, } s_{1}=\text { very poor, } s_{2}=\text { poor } \\
s_{3} & =\text { slightly poor, } s_{4}=\text { fair, } s_{5}=\text { slightly good } \\
s_{6} & \left.=\text { good, } s_{7}=\text { very good, } s_{8}=\text { extremely good }\right\} .
\end{aligned}
$$

All the LPFNs can form an LPFDM $R=\left(r_{i j}\right)_{4 \times 4}$ as shown in Table 2. 
TABLE 1: The LPFDM for the selection of firewall productions.

\begin{tabular}{lcccc}
\hline & $u_{1}$ & $u_{2}$ & $u_{3}$ & $u_{4}$ \\
\hline$c_{1}$ & $\left(s_{4}, s_{2}\right)$ & $\left(s_{7}, s_{1}\right)$ & $\left(s_{5}, s_{2}\right)$ & $\left(s_{6}, s_{2}\right)$ \\
$c_{2}$ & $\left(s_{4}, s_{3}\right)$ & $\left(s_{6}, s_{1}\right)$ & $\left(s_{5}, s_{1}\right)$ & $\left(s_{5}, s_{3}\right)$ \\
$c_{3}$ & $\left(s_{5}, s_{1}\right)$ & $\left(s_{6}, s_{2}\right)$ & $\left(s_{6}, s_{2}\right)$ & $\left(s_{5}, s_{4}\right)$ \\
$c_{4}$ & $\left(s_{6}, s_{2}\right)$ & $\left(s_{5}, s_{3}\right)$ & $\left(s_{5}, s_{1}\right)$ & $\left(s_{6}, s_{1}\right)$ \\
$c_{5}$ & $\left(s_{7}, s_{1}\right)$ & $\left(s_{6}, s_{1}\right)$ & $\left(s_{7}, s_{1}\right)$ & $\left(s_{6}, s_{2}\right)$ \\
\hline
\end{tabular}

TABle 2: The LPFDM for the security evaluation of computer systems.

\begin{tabular}{ccccc}
\hline & $u_{1}$ & $u_{2}$ & $u_{3}$ & $u_{4}$ \\
\hline$c_{1}$ & $\left(s_{5}, s_{3}\right)$ & $\left(s_{7}, s_{1}\right)$ & $\left(s_{5}, s_{2}\right)$ & $\left(s_{6}, s_{2}\right)$ \\
$c_{2}$ & $\left(s_{7}, s_{1}\right)$ & $\left(s_{7}, s_{3}\right)$ & $\left(s_{6}, s_{2}\right)$ & $\left(s_{6}, s_{2}\right)$ \\
$c_{3}$ & $\left(s_{6}, s_{2}\right)$ & $\left(s_{5}, s_{1}\right)$ & $\left(s_{6}, s_{2}\right)$ & $\left(s_{6}, s_{1}\right)$ \\
$c_{4}$ & $\left(s_{6}, s_{1}\right)$ & $\left(s_{6}, s_{2}\right)$ & $\left(s_{6}, s_{1}\right)$ & $\left(s_{5}, s_{1}\right)$ \\
\hline
\end{tabular}

5.2.1. The Proposed LPF-TOPSIS Method. The proposed LPF-TOPSIS method is used to handle Example 2, which involves the following steps:

Step 1: since all the attributes are benefit-type, it is unnecessary to normalize the LPFDM $R=\left(r_{i j}\right)_{4 \times 4}$.

Step 2: using equation (30), the weights of the attributes can be computed as

$$
\omega=(0.2681,0.3068,0.2088,0.2163) \text {. }
$$

Step 3: according to the LPFDM, the linguistic Pythagorean fuzzy PIS and NIS can be obtained as

$$
\begin{aligned}
& R^{+}=\left[\left(s_{7}, s_{1}\right),\left(s_{7}, s_{1}\right),\left(s_{6}, s_{1}\right),\left(s_{6}, s_{1}\right)\right], \\
& R^{-}=\left[\left(s_{5}, s_{3}\right),\left(s_{5}, s_{3}\right),\left(s_{5}, s_{2}\right),\left(s_{5}, s_{2}\right)\right] .
\end{aligned}
$$

Step 4: using equation (34), the weighted correlation coefficient between each alternative and the linguistic Pythagorean fuzzy PIS is computed as

$$
\begin{aligned}
& K_{1}^{+}\left(r_{1 j}, R^{+}\right)=0.9377, \\
& K_{2}^{+}\left(r_{2 j}, R^{+}\right)=0.9900, \\
& K_{3}^{+}\left(r_{3 j}, R^{+}\right)=0.9038, \\
& K_{4}^{+}\left(r_{4 j}, R^{+}\right)=0.9508 .
\end{aligned}
$$

Using equation (35), the weighted correlation coefficient between each alternative and the linguistic Pythagorean fuzzy NIS is computed as

$$
\begin{aligned}
& K_{1}^{-}\left(r_{1 j}, R^{-}\right)=0.9050, \\
& K_{2}^{-}\left(r_{2 j}, R^{-}\right)=0.8221, \\
& K_{3}^{-}\left(r_{3 j}, R^{-}\right)=0.9534, \\
& K_{4}^{-}\left(r_{4 j}, R^{-}\right)=0.9616 .
\end{aligned}
$$

Step 5: using equation (36), the relative closeness of each alternative is calculated as

$$
\begin{aligned}
& \eta_{1}=0.5089 \\
& \eta_{2}=0.5463 \\
& \eta_{3}=0.4866 \\
& \eta_{4}=0.4972
\end{aligned}
$$

Step 6: According to the values of relative closeness, all the alternatives can be ranked as

$$
c_{2}>c_{1}>c_{4}>c_{3} \text {. }
$$

Step 7: end.

5.2.2. The TOPSIS Method Based on Distance Measure. The TOPSIS method based on distance measure computes the relative closeness values of alternatives using the distance measure between each alternative and ideal solution. According to the literature [63], we first propose the distance measure for LPFSs.

Definition 7. Given a finite reference set $X=\left\{x_{1}, x_{2}, \ldots, x_{n}\right\}$ and a continuous LTS $S=\left\{s_{\beta} \mid \beta \in[0, \tau]\right\}$, if $A=\left\{\left\langle x_{i}, s_{p_{A}}\right.\right.$ $\left.\left.\left(x_{i}\right), s_{q_{A}}\left(x_{i}\right)\right\rangle \mid x_{i} \in X\right\}$ and $B=\left\{\left\langle x_{i}, s_{p_{B}}\left(x_{i}\right), s_{q_{B}}\left(x_{i}\right)\right\rangle \mid x_{i} \in\right.$ $X\}$ are two LPFSs on $X$, then the distance measure between them is defined as

$$
\begin{aligned}
d(A, B)= & \frac{1}{2 \tau^{2}} \sum_{j=1}^{n} \omega_{j} *\left(\left|\left(I\left(s_{p_{A}}\left(x_{i}\right)\right)\right)^{2}-\left(I\left(s_{p_{B}}\left(x_{i}\right)\right)\right)^{2}\right|\right. \\
& +\left|\left(I\left(s_{q_{A}}\left(x_{i}\right)\right)\right)^{2}-\left(I\left(s_{q_{B}}\left(x_{i}\right)\right)\right)^{2}\right| \\
& \left.+\left|\left(I\left(s_{h_{A}}\left(x_{i}\right)\right)\right)^{2}-\left(I\left(s_{h_{B}}\left(x_{i}\right)\right)\right)^{2}\right|\right) .
\end{aligned}
$$

Based on the distance measure, the relative closeness of each alternative can be calculated as

$$
\eta_{i}=\frac{d_{i}^{-}\left(r_{i j}, R^{-}\right)}{d_{i}^{+}\left(r_{i j}, R^{+}\right)+d_{i}^{-}\left(r_{i j}, R^{-}\right)},
$$

where $d_{i}^{+}\left(r_{i j}, R^{+}\right)$and $d_{i}^{-}\left(r_{i j}, R^{-}\right)$are the distance measure between the alternative and PIS and distance measure between the alternative and NIS, respectively.

The decision-making process of the TOPSIS method based on distance measure is given below:

Steps 1-3: see Steps 1-3 of the proposed LPF-TOPSIS method.

Step 4: using equation (51), the distance measure between each alternative and the linguistic Pythagorean fuzzy PIS is computed as 


$$
\begin{aligned}
& d_{1}^{+}\left(r_{1 j}, R^{+}\right)=0.0795, \\
& d_{2}^{+}\left(r_{2 j}, R^{+}\right)=0.0485, \\
& d_{3}^{+}\left(r_{3 j}, R^{+}\right)=0.1652, \\
& d_{4}^{+}\left(r_{4 j}, R^{+}\right)=0.1540,
\end{aligned}
$$

and the distance measure between each alternative and the linguistic Pythagorean fuzzy NIS is computed as

$$
\begin{aligned}
& d_{1}^{-}\left(r_{1 j}, R^{-}\right)=0.1522, \\
& d_{2}^{-}\left(r_{2 j}, R^{-}\right)=0.3078, \\
& d_{3}^{-}\left(r_{3 j}, R^{-}\right)=0.1575, \\
& d_{4}^{-}\left(r_{4 j}, R^{-}\right)=0.1448 .
\end{aligned}
$$

Step 5: using equation (52), the relative closeness of each alternative is computed as

$$
\begin{aligned}
& \eta_{1}=0.6567, \\
& \eta_{2}=0.8639, \\
& \eta_{3}=0.4881, \\
& \eta_{4}=0.4846 .
\end{aligned}
$$

Step 6: according to the values of relative closeness, all the alternatives can be ranked as

$$
c_{2}>c_{1}>c_{3}>c_{4}
$$

Step 7: end.

5.2.3. The Existing Decision-Making Methods Based on LPFWA and LPFWG Operators [41]. The existing decisionmaking methods based on LPFWA and LPFWG operators [41] are used to solve Example 2:

Steps 1-2: see Steps 1-2 of the proposed LPF-TOPSIS method.

Step 3: use the LPFWA defined in equation (2) to aggregate the attribute values $r_{i j}, j=1,2,3,4$ of each alternative as

$$
\begin{aligned}
& \operatorname{LPFWA}\left(r_{11}, r_{12}, r_{13}, r_{14}\right)=\left(s_{6.0763}, s_{1.8025}\right), \\
& \operatorname{LPFWA}\left(r_{21}, r_{22}, r_{23}, r_{24}\right)=\left(s_{6.6665}, s_{1.8808}\right), \\
& \operatorname{LPFWA}\left(r_{31}, r_{32}, r_{33}, r_{34}\right)=\left(s_{5.7449}, s_{1.3917}\right), \\
& \operatorname{LPFWA}\left(r_{41}, r_{42}, r_{43}, r_{44}\right)=\left(s_{5.8240}, s_{1.2370}\right),
\end{aligned}
$$

and use the LPFWG defined in equation (3) to fuse the attribute values $r_{i j}, j=1,2,3,4$ of each alternative as

$$
\begin{aligned}
& \operatorname{LPFWG}\left(r_{11}, r_{12}, r_{13}, r_{14}\right)=\left(s_{5.7667}, s_{2.1212}\right), \\
& \operatorname{LPFWG}\left(r_{21}, r_{22}, r_{23}, r_{24}\right)=\left(s_{6.5560}, s_{2.1935}\right), \\
& \operatorname{LPFWG}\left(r_{31}, r_{32}, r_{33}, r_{34}\right)=\left(s_{5.6736}, s_{1.5649}\right), \\
& \operatorname{LPFWG}\left(r_{41}, r_{42}, r_{43}, r_{44}\right)=\left(s_{5.7680}, s_{1.3914}\right) .
\end{aligned}
$$

Step 4: use Definition 2 to compute the score function value of each alternative for the LPFWA as

$$
\begin{aligned}
& S\left(c_{1}\right)=s_{6.9883}, \\
& S\left(c_{2}\right)=s_{7.2424}, \\
& S\left(c_{3}\right)=s_{6.8945}, \\
& S\left(c_{4}\right)=s_{6.9422},
\end{aligned}
$$

and the score value of each alternative for the LPFWG as

$$
\begin{aligned}
& S\left(c_{1}\right)=s_{6.8101}, \\
& S\left(c_{2}\right)=s_{7.1474}, \\
& S\left(c_{3}\right)=s_{6.8462}, \\
& S\left(c_{4}\right)=s_{6.9041} .
\end{aligned}
$$

Step 5: according to the score function values, all the alternatives can be ranked as

$$
c_{2}>c_{1}>c_{4}>c_{3}
$$

when the LPFWA operator is used.

$$
c_{2}>c_{4}>c_{3}>c_{1}
$$

when the LPFWG operator is used.

Step 6: end.

5.2.4. The Existing Decision-Making Methods Based on LPFWIPBM and LPFWIPGBM Operators [42]. We use the existing decision-making methods based on LPFWIPBM and LPFWIPGBM operators [42] to handle Example 2:

Steps 1-2: see Steps 1-2 of the proposed LPF-TOPSIS method.

Step 3: use the LPFWIPBM operator defined in [42] to aggregate the attribute values $r_{i j}, j=1,2,3,4$ of each alternative as

$$
\begin{aligned}
& \operatorname{LPFWIPBM}^{p, q}\left(r_{11}, r_{12}, r_{13}, r_{14}\right)=\left(s_{3.5382}, s_{0.7939}\right), \\
& \operatorname{LPFWIPBM}^{p, q}\left(r_{21}, r_{22}, r_{23}, r_{24}\right)=\left(s_{4.2213}, s_{1.6548}\right), \\
& \operatorname{LPFWIPBM}^{p, q}\left(r_{31}, r_{32}, r_{33}, r_{34}\right)=\left(s_{3.3287}, s_{0.8415}\right), \\
& \operatorname{LPFWIPBM}^{p, q}\left(r_{41}, r_{42}, r_{43}, r_{44}\right)=\left(s_{3.3732}, s_{0.7435}\right),
\end{aligned}
$$

and use the LPFWIPGBM operator defined in [42] to aggregate the attribute values $r_{i j}, j=1,2,3,4$ of each alternative as 


$$
\begin{aligned}
& \operatorname{LPFWIPGBM}^{p, q}\left(r_{11}, r_{12}, r_{13}, r_{14}\right)=\left(s_{2.5388}, s_{0.7119}\right), \\
& \operatorname{LPFWIPGBM}^{p, q}\left(r_{21}, r_{22}, r_{23}, r_{24}\right)=\left(s_{3.2710}, s_{0.7663}\right), \\
& \operatorname{LPFWIPGBM}^{p, q}\left(r_{31}, r_{32}, r_{33}, r_{34}\right)=\left(s_{2.4215}, s_{0.5747}\right), \\
& \operatorname{LPFWIPGBM}^{p, q}\left(r_{41}, r_{42}, r_{43}, r_{44}\right)=\left(s_{2.4893}, s_{0.4752}\right) .
\end{aligned}
$$

Step 4: use Definition 2 to compute the score function value of each alternative for the LPFWIPBM operator as

$$
\begin{aligned}
& S\left(c_{1}\right)=s_{6.1599}, \\
& S\left(c_{2}\right)=s_{6.2891}, \\
& S\left(c_{3}\right)=s_{6.0980}, \\
& S\left(c_{4}\right)=s_{6.1165},
\end{aligned}
$$

and the score function value of each alternative for the LPFWIPGBM operator as

$$
\begin{aligned}
& S\left(c_{1}\right)=s_{5.9135}, \\
& S\left(c_{2}\right)=s_{6.0874}, \\
& S\left(c_{3}\right)=s_{5.8965}, \\
& S\left(c_{4}\right)=s_{5.9148} .
\end{aligned}
$$

Step 5: according to the score function values, all the alternatives can be ranked as

$$
c_{2}>c_{1}>c_{4}>c_{3}
$$

when the LPFWIPBM operator is used.

$$
c_{2}>c_{4}>c_{1}>c_{3}
$$

when the LPFWIPGBM operator is used.

Step 6: end.

The ranking results of the proposed LPF-TOPSIS method and existing decision-making methods [41, 42] are summarized as shown in Table 3.

From Table 3, it can be noted that all the decision-making methods obtain the same best solution, and the proposed LPFTOPSIS method and the existing decision-making methods based on LPFWA and LPFWIPBM operators derive the same

\begin{tabular}{|c|c|}
\hline Methods & $\begin{array}{l}\text { Ranking } \\
\text { results }\end{array}$ \\
\hline The proposed LPF-TOPSIS 1 & $c_{2}>c_{1}>c_{4}>c_{3}$ \\
\hline The TOPSIS method based on distance measure & $c_{2}>c_{1}>c_{3}>c_{4}$ \\
\hline $\begin{array}{l}\text { The existing decision-making method based on } \\
\text { LPFWA [41] }\end{array}$ & $c_{2}>c_{1}>c_{4}>c_{3}$ \\
\hline $\begin{array}{l}\text { The existing decision-making method based on } \\
\text { LPFWG [41] }\end{array}$ & $c_{2}>c_{4}>c_{3}>c_{1}$ \\
\hline $\begin{array}{l}\text { The existing decision-making method based on } \\
\text { LPFWIPBM [42] }\end{array}$ & $c_{2}>c_{1}>c_{4}>c_{3}$ \\
\hline $\begin{array}{l}\text { The existing decision-making method based on } \\
\text { LPFWIPGBM [42] }\end{array}$ & $c_{2}>c_{4}>c_{1}>c_{3}$ \\
\hline
\end{tabular}
ranking result. It indicates that the proposed LPF-TOPSIS method is practicable. However, the proposed LPF-TOPSIS method shows different ranking results from the TOPSIS method based on distance measure and existing decisionmaking methods based on LPFWG and LPFWIPGBM operators. The reasons can be analyzed as follows:

(1) The proposed LPF-TOPSIS method can efficiently achieve trade-offs among conflicting attributes, which usually exist in the MADM problems

(2) In Example 2, the correlation coefficient performs better than the distance measure when they are used to distinguish ideal solutions
TABLE 3: Ranking results for various decision-making methods.

\section{Conclusions}

In this paper, a novel LPF-TOPSIS method is proposed to solve the MCDM problems under the LPFS environment. The correlation coefficient measure is used to replace the distance measure so as to improve the decision results of the LPFTOPSIS method. Two entropy measures are also put forward to measure the uncertainty degree of LPFSs, based on which, the attribute weight information can be derived. A case study concerning the selection of firewall productions is provided to demonstrate the application of the proposed LPF-TOPSIS method. The comparative analyses can test the effectiveness and advantages of the proposed LPF-TOPSIS method.

The advantages of our study are summarized as follows:

(1) The existing decision-making methods based on aggregation operators $[41,42]$ simply aggregate a series of LPFSs into a single one. They do not consider the relationships among attributes. By contrast, our proposed LPF-TOPSIS method can efficiently achieve trade-offs among conflicting attributes.

(2) In this study, two novel entropy measures are proposed to determine the unknown attribute weight information. By contrast, the attribute weight information in the studies $[41,42]$ is provided by DMs in advance.

(3) Compared with the TOPSIS method based on distance measure, the proposed LPF-TOPSIS method uses the correlation coefficient to measure the proximity between each alternative and ideal solution. It is an innovative way to make the decisions under the LPFS environment.

However, our study still has some limitations as follows:

(1) It is insufficient to only consider the evaluation information from individual DMs when making the decision results

(2) In our proposed LPF-TOPSIS method, the attribute weight information determining method is an objective method, which neglects the preference information of DMs

In the future, we will apply the concept of LPFSs into the group MADM problems. Moreover, various decisionmaking methods will be extended to handle LPFSs. 


\section{Data Availability}

The data used to support the findings of this study are included within the article.

\section{Conflicts of Interest}

The authors declare that they have no conflicts of interest.

\section{Acknowledgments}

This research work was supported by the National Natural Science Foundation of China under Grant nos. 61872086, U1805263, and 61672157 and Distinguished Young Scientific Research Talents Plan in Universities of Fujian Province (2017).

\section{References}

[1] H. Garg, "Some methods for strategic decision-making problems with immediate probabilities in Pythagorean fuzzy environment," International Journal of Intelligent Systems, vol. 33, no. 4, pp. 687-712, 2018.

[2] M. Lin, Z. Xu, Y. Zhai, and Z. Yao, "Multi-attribute group decision-making under probabilistic uncertain linguistic environment," Journal of the Operational Research Society, vol. 69, no. 2, pp. 157-170, 2018.

[3] M. Lin, Z. Chen, H. Liao, and Z. Xu, "ELECTRE II method to deal with probabilistic linguistic term sets and its application to edge computing," Nonlinear Dynamics, vol. 96, no. 3, pp. 2125-2143, 2019.

[4] M. Lin, Q. Zhan, Z. Xu, and R. Chen, "Group decision-making model with hesitant multiplicative preference relations based on regression method and feedback mechanism," IEEE Access, vol. 6, pp. 61130-61150, 2018.

[5] M. Lin, Q. Zhan, Z. Xu, and R. Chen, "Group decision making with probabilistic hesitant multiplicative preference relations based on consistency and consensus," IEEE Access, vol. 6, pp. 63329-63344, 2018.

[6] G. Wei, C. Wei, J. Wang, H. Gao, and Y. Wei, "Some q-rung orthopair fuzzy maclaurin symmetric mean operators and their applications to potential evaluation of emerging technology commercialization," International Journal of Intelligent Systems, vol. 34, no. 1, pp. 50-81, 2019.

[7] J. B. Xiong, X. H. Chen, Q. Yang, L. Chen, and Z. Q. Yao, “A task-oriented user selection incentive mechanism in edgeaided mobile crowdsensing," IEEE Transactions on Network Science and Engineering, 2019.

[8] J. Xiong, J. Ren, L. Chen et al., "Enhancing privacy and availability for data clustering in intelligent electrical service of IoT," IEEE Internet of Things Journal, vol. 6, no. 2, pp. 1530-1540, 2019.

[9] H. Garg, "Analysis of an industrial system under uncertain environment by using different types of fuzzy numbers," International Journal of System Assurance Engineering and Management, vol. 9, no. 2, pp. 525-538, 2018.

[10] R. R. Yager, N. Alajlan, and Y. Bazi, "Aspects of generalized orthopair fuzzy sets," International Journal of Intelligent Systems, vol. 33, no. 11, pp. 2154-2174, 2018.

[11] Z. Xu and H. Wang, "On the syntax and semantics of virtual linguistic terms for information fusion in decision making," Information Fusion, vol. 34, pp. 43-48, 2017.
[12] M. S. Yang and Z. Hussain, "Fuzzy entropy for pythagorean fuzzy sets with application to multicriterion decision making," Complexity, vol. 2018, Article ID 2832839, 14 pages, 2018.

[13] A. Mahdiyar, S. Tabatabaee, S. Durdyev, S. Ismail, A. Abdullah, and W. N. M. Wan Mohd Rani, "A prototype decision support system for green roof type selection: a cybernetic fuzzy ANP method," Sustainable Cities and Society, vol. 48, p. 101532, 2019.

[14] L. A. Zadeh, "Fuzzy sets," Information and Control, vol. 8, no. 3, pp. 338-353, 1965.

[15] K. T. Atanassov, "Intuitionistic fuzzy sets," Fuzzy Sets and Systems, vol. 20, no. 1, pp. 87-96, 1986.

[16] R. R. Yager and A. M. Abbasov, "Pythagorean membership grades, complex numbers, and decision making," International Journal of Intelligent Systems, vol. 28, no. 5, pp. 436-452, 2013.

[17] R. R. Yager, "Generalized orthopair fuzzy sets," IEEE Transactions on Fuzzy Systems, vol. 25, no. 5, pp. 1222-1230, 2017.

[18] X. Peng, H. Yuan, and Y. Yang, "Pythagorean fuzzy information measures and their applications," International Journal of Intelligent Systems, vol. 32, no. 10, pp. 991-1029, 2017.

[19] H. Garg, "Novel neutrality operation-based Pythagorean fuzzy geometric aggregation operators for multiple attribute group decision analysis," International Journal of Intelligent Systems, vol. 34, no. 10, pp. 2459-2489, 2019.

[20] H. Garg, "New logarithmic operational laws and their aggregation operators for Pythagorean fuzzy set and their applications," International Journal of Intelligent Systems, vol. 34, no. 1, pp. 82-106, 2019.

[21] H. Garg, "New exponential operational laws and their aggregation operators for interval-valued Pythagorean fuzzy multicriteria decision-making," International Journal of Intelligent Systems, vol. 33, no. 3, pp. 653-683, 2018.

[22] H. Garg, "Hesitant Pythagorean fuzzy Maclaurin symmetric mean operators and its applications to multiattribute decision-making process," International Journal of Intelligent Systems, vol. 34, no. 4, pp. 601-626, 2019.

[23] X. D. Peng and H. Garg, "Multiparametric similarity measures on pythagorean fuzzy sets with applications to pattern recognition," Applied Intelligence, 2019.

[24] H. Garg, "A novel correlation coefficients between Pythagorean fuzzy sets and its applications to decision-making processes," International Journal of Intelligent Systems, vol. 31, no. 12, pp. 1234-1252, 2016.

[25] F. Xiao and W. Ding, "Divergence measure of Pythagorean fuzzy sets and its application in medical diagnosis," Applied Soft Computing, vol. 79, pp. 254-267, 2019.

[26] G. Wei and Y. Wei, "Similarity measures of Pythagorean fuzzy sets based on the cosine function and their applications," International Journal of Intelligent Systems, vol. 33, no. 3, pp. 634-652, 2018.

[27] T.-Y. Chen, “An outranking approach using a risk attitudinal assignment model involving Pythagorean fuzzy information and its application to financial decision making," Applied Soft Computing, vol. 71, pp. 460-487, 2018.

[28] C. Zhang, D. Li, Y. Mu, and D. Song, "A Pythagorean fuzzy multigranulation probabilistic model for mine ventilator fault diagnosis," Complexity, vol. 2018, Article ID 7125931, 19 pages, 2018.

[29] X. Gou and Z. Xu, "Novel basic operational laws for linguistic terms, hesitant fuzzy linguistic term sets and probabilistic 
linguistic term sets," Information Sciences, vol. 372, pp. 407427, 2016.

[30] H. Garg and K. Kumar, "Linguistic interval-valued Atanassov intuitionistic fuzzy sets and their applications to group decision-making problems," IEEE Transactions on Fuzzy Systems, 2019.

[31] R. Arora and H. Garg, "Group decision-making method based on prioritized linguistic intuitionistic fuzzy aggregation operators and its fundamental properties," Computational and Applied Mathematics, vol. 38, no. 2, pp. 1-36, 2019.

[32] H. Garg and K. Kumar, "Multiattribute decision making based on power operators for linguistic intuitionistic fuzzy set using set pair analysis," Expert Systems, vol. 36, no. 4, Article ID e12428, 2019.

[33] H. Garg and Nancy, "Algorithms for possibility linguistic single-valued neutrosophic decision-making based on COPRAS and aggregation operators with new information measures," Measurement, vol. 138, pp. 278-290, 2019.

[34] L. A. Zadeh, "The concept of a linguistic variable and its application to approximate reasoning-I," Information Sciences, vol. 8, no. 3, pp. 199-249, 1975.

[35] Q. Pang, H. Wang, and Z. Xu, "Probabilistic linguistic term sets in multi-attribute group decision making," Information Sciences, vol. 369, pp. 128-143, 2016.

[36] Y. Wu and J. Zhou, "Risk assessment of urban rooftop distributed PV in energy performance contracting (EPC) projects: an extended HFLTS-DEMATEL fuzzy synthetic evaluation analysis," Sustainable Cities and Society, vol. 47, Article ID 101524, 2019.

[37] H. Zhang, "Linguistic intuitionistic fuzzy sets and application in MAGDM," Journal of Applied Mathematics, vol. 2014, Article ID 432092, 11 pages, 2014.

[38] Z. S. Xu, Linguistic Decision Making: Theory and Methods, Springer, Berlin, Germany, 2012.

[39] X. Wu, H. Liao, Z. Xu, A. Hafezalkotob, and F. Herrera, "Probabilistic linguistic MULTIMOORA: a multicriteria decision making method based on the probabilistic linguistic Expectation function and the improved Borda rule," IEEE Transactions on Fuzzy Systems, vol. 26, no. 6, pp. 3688-3702, 2018.

[40] H. Liao, X. Wu, X. Liang, J. Xu, and F. Herrera, “A new hesitant fuzzy linguistic ORESTE method for hybrid multicriteria decision making," IEEE Transactions on Fuzzy Systems, vol. 26, no. 6, pp. 3793-3807, 2018.

[41] H. Garg, "Linguistic Pythagorean fuzzy sets and its applications in multiattribute decision-making process," International Journal of Intelligent Systems, vol. 33, no. 6, pp. 1234-1263, 2018.

[42] M. W. Lin, J. H. Wei, Z. S. Xu, and R. Q. Chen, "Multiattribute group decision-making based on linguistic pythagorean fuzzy interaction partitioned bonferroni mean aggregation operators," Complexity, vol. 2018, Article ID 9531064, 24 pages, 2018.

[43] M. Akram, W. A. Dudek, and F. Ilyas, "Group decisionmaking based on pythagorean fuzzy TOPSIS method," International Journal of Intelligent Systems, vol. 34, no. 7, pp. 1455-1475, 2019.

[44] C. L. Hwang and K. Yoon, Multiple Attribute Decision Making: Methods and Applications, Springer-Verlag, Berlin, Germany, 1981.

[45] S. Opricovic and G.-H. Tzeng, "Compromise solution by MCDM methods: a comparative analysis of VIKOR and TOPSIS," European Journal of Operational Research, vol. 156, no. 2, pp. 445-455, 2004.
[46] K. Kumar and H. Garg, "Connection number of set pair analysis based TOPSIS method on intuitionistic fuzzy sets and their application to decision making," Applied Intelligence, vol. 48, no. 8, pp. 2112-2119, 2018.

[47] K. Kumar and H. Garg, "TOPSIS method based on the connection number of set pair analysis under interval-valued intuitionistic fuzzy set environment," Computational and Applied Mathematics, vol. 37, no. 2, pp. 1319-1329, 2018.

[48] H. Garg and K. Kumar, "A novel exponential distance and its based topsis method for interval-valued intuitionistic fuzzy sets using connection number of spa theory," Artificial Intelligence Review, 2018.

[49] X. Zhang and Z. Xu, "Extension of TOPSIS to multiple criteria decision making with Pythagorean fuzzy sets," International Journal of Intelligent Systems, vol. 29, no. 12, pp. 1061-1078, 2014.

[50] D. Liang, Z. Xu, D. Liu, and Y. Wu, "Method for three-way decisions using ideal TOPSIS solutions at Pythagorean fuzzy information," Information Sciences, vol. 435, pp. 282-295, 2018.

[51] A. Biswas and B. Sarkar, "Pythagorean fuzzy TOPSIS for multicriteria group decision-making with unknown weight information through entropy measure," International Journal of Intelligent Systems, vol. 34, no. 6, pp. 1108-1128, 2019.

[52] K. Yoon, "A reconciliation among discrete compromise solutions," The Journal of the Operational Research Society, vol. 38, no. 3, pp. 277-286, 1987.

[53] G. Sun, X. Guan, X. Yi, and Z. Zhou, "An innovative TOPSIS approach based on hesitant fuzzy correlation coefficient and its applications," Applied Soft Computing, vol. 68, pp. 249267, 2018.

[54] C.-L. Hwang, Y.-J. Lai, and T.-Y. Liu, “A new approach for multiple objective decision making," Computers \& Operations Research, vol. 20, no. 8, pp. 889-899, 1993.

[55] H. Garg, "A new improved score function of an intervalvalued Pythagorean fuzzy set based TOPSIS method," International Journal for Uncertainty Quantification, vol. 7, no. 5, pp. 463-474, 2017.

[56] X. Zhang, "A novel probabilistic linguistic approach for largescale group decision making with incomplete weight information," International Journal of Fuzzy Systems, vol. 20, no. 7, pp. 2245-2256, 2018.

[57] J. Ye, "Multicriteria fuzzy decision-making method using entropy weights-based correlation coefficients of intervalvalued intuitionistic fuzzy sets," Applied Mathematical Modelling, vol. 34, no. 12, pp. 3864-3870, 2010.

[58] M. Xia and Z. Xu, "Entropy/cross entropy-based group decision making under intuitionistic fuzzy environment," Information Fusion, vol. 13, no. 1, pp. 31-47, 2012.

[59] Z. Xu and M. Xia, "Hesitant fuzzy entropy and cross-entropy and their use in multiattribute decision-making," International Journal of Intelligent Systems, vol. 27, no. 9, pp. 799-822, 2012.

[60] Z. Trabelsi, S. Zeidan, K. Shuaib, and K. Salah, "Improved session table architecture for denial of stateful firewall attacks," IEEE Access, vol. 6, pp. 35528-35543, 2018.

[61] N. K. Sreelaja and G. A. Vijayalakshmi Pai, "Ant colony optimization based approach for efficient packet filtering in firewall," Applied Soft Computing, vol. 10, no. 4, pp. 12221236, 2010.

[62] C. Gan, "Modeling and analysis of the effect of network eigenvalue on viral spread," Nonlinear Dynamics, vol. 84, no. 3 , pp. 1727-1733, 2016.

[63] X. Peng and G. Selvachandran, "Pythagorean fuzzy set: state of the art and future directions," Artificial Intelligence Review, vol. 52, no. 3, pp. 1873-1927, 2019. 


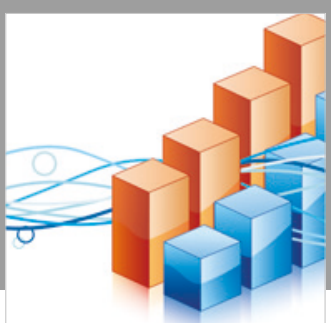

Advances in

Operations Research

\section{-n-m}
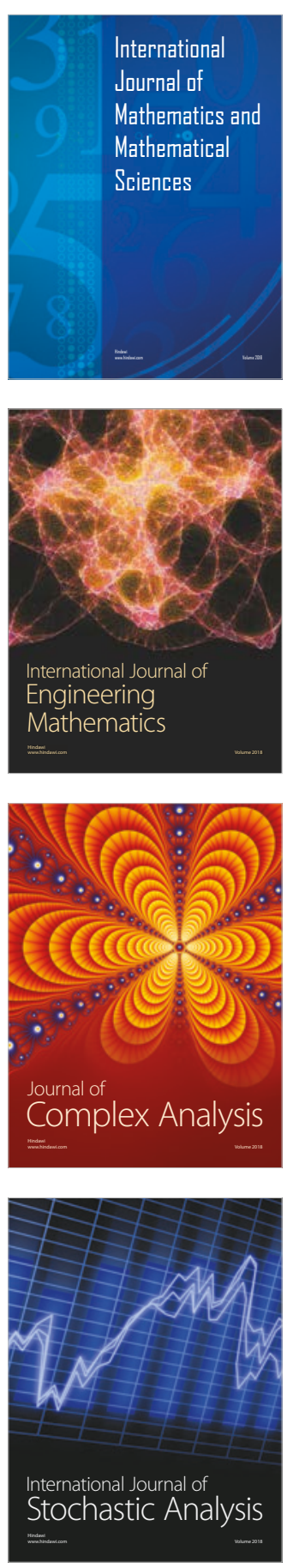
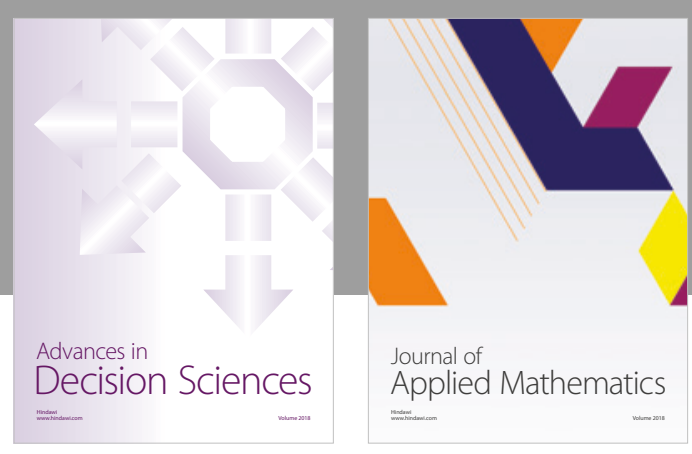

Journal of

Applied Mathematics
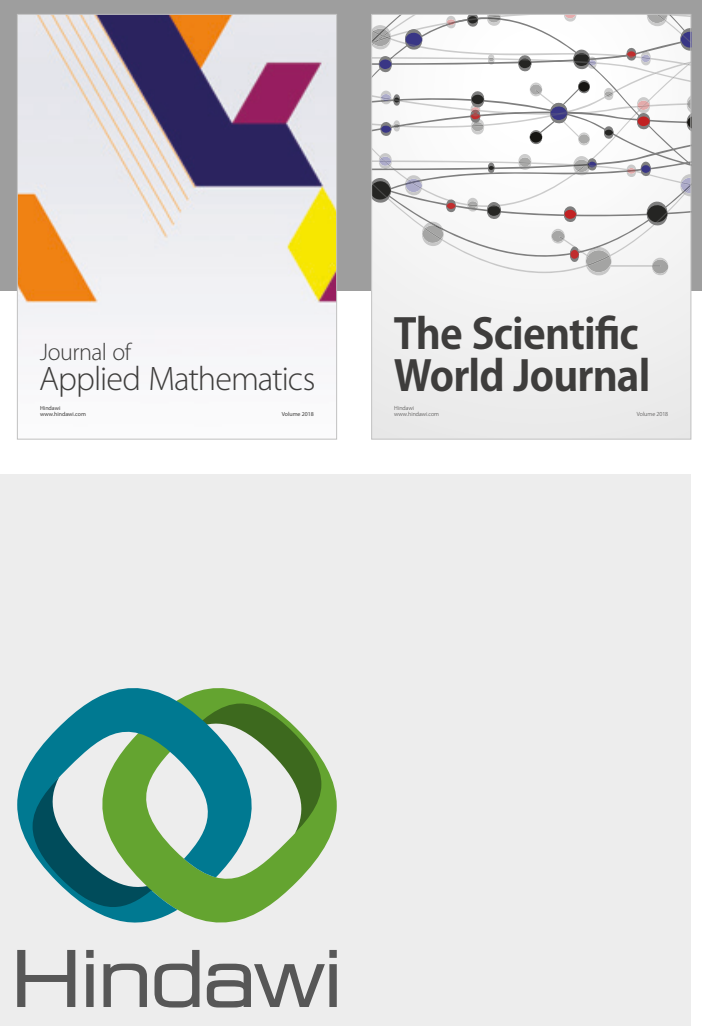

Submit your manuscripts at

www.hindawi.com

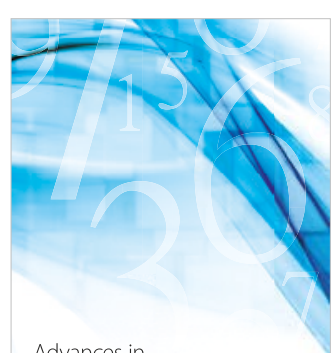

Advances in
Numerical Analysis
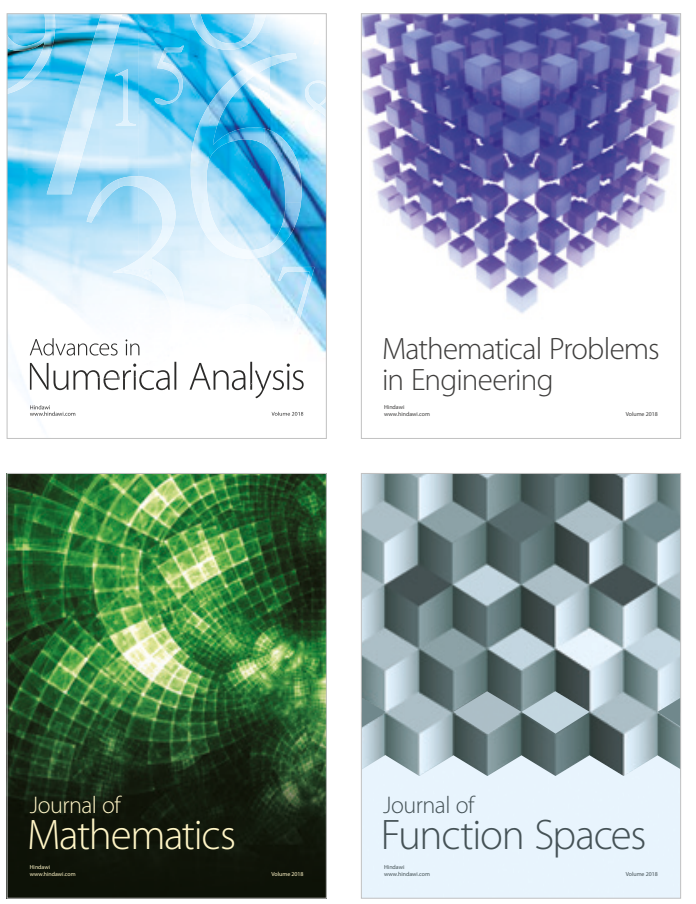

Mathematical Problems in Engineering

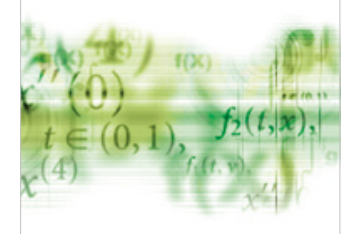

International Journal of

Differential Equations

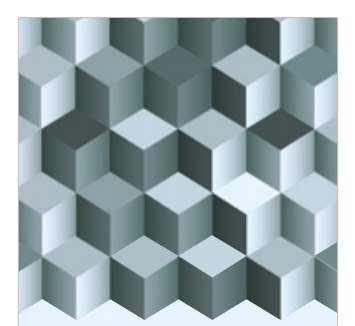

Journal of

Function Spaces
The Scientific

World Journal

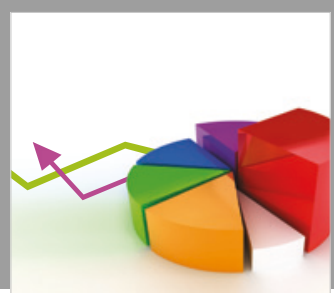

Journal of

Probability and Statistics
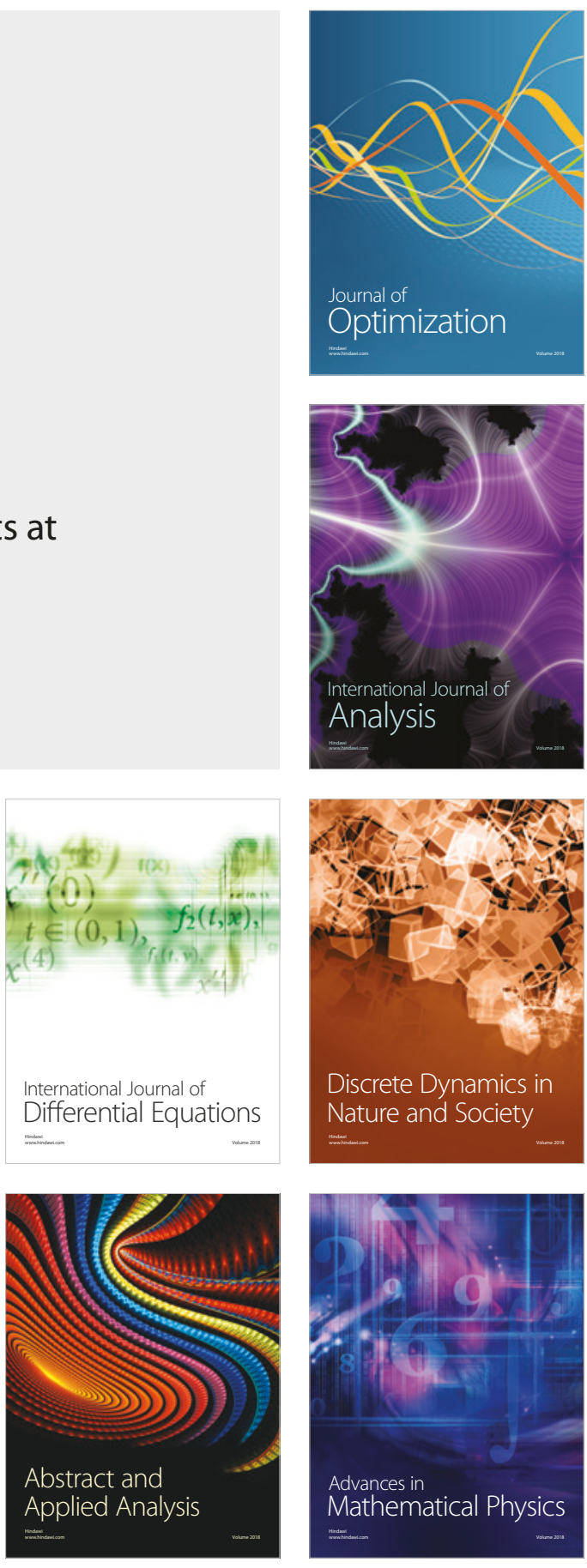